\title{
Geometrically Induced Force Interaction for Three-Dimensional Deformable Models
}

\author{
Si Yong Yeo, Xianghua Xie, Member, IEEE, Igor Sazonov, and Perumal Nithiarasu
}

\begin{abstract}
In this paper, we propose a novel 3-D deformable model that is based upon a geometrically induced external force field which can be conveniently generalized to arbitrary dimensions. This external force field is based upon hypothesized interactions between the relative geometries of the deformable model and the object boundary characterized by image gradient. The evolution of the deformable model is solved using the level set method so that topological changes are handled automatically. The relative geometrical configurations between the deformable model and the object boundaries contribute to a dynamic vector force field that changes accordingly as the deformable model evolves. The geometrically induced dynamic interaction force has been shown to greatly improve the deformable model performance in acquiring complex geometries and highly concave boundaries, and it gives the deformable model a high invariancy in initialization configurations. The voxel interactions across the whole image domain provide a global view of the object boundary representation, giving the external force a long attraction range. The bidirectionality of the external force field allows the new deformable model to deal with arbitrary cross-boundary initializations, and facilitates the handling of weak edges and broken boundaries. In addition, we show that by enhancing the geometrical interaction field with a nonlocal edge-preserving algorithm, the new deformable model can effectively overcome image noise. We provide a comparative study on the segmentation of various geometries with different topologies from both synthetic and real images, and show that the proposed method achieves significant improvements against existing image gradient techniques.
\end{abstract}

Index Terms - Deformable model, geometric potential field, level set method, object localization, 3-D segmentation.

\section{INTRODUCTION}

$\mathbf{S}$ HAPE segmentation from volumetric data has an important role in applications such as medical image analysis. Volumetric image segmentation remains an intricate process, due to the complexity and variability of image data and shapes (i.e., anatomical structures).

There have been applications of simple techniques such as thresholding and region growing in the extraction of 3-D objects

Manuscript received December 29, 2009; revised September 13, 2010; accepted October 28, 2010. Date of publication November 15, 2010; date of current version April 15, 2011. This work was supported in part by EPSRC D070554 and Research Council UK Academic Fellowship. The associate editor coordinating the review of this manuscript and approving it for publication was Dr. Arun Ross.

S. Y. Yeo, I. Sazonov, and P. Nithiarasu are with the College of Engineering, Swansea University, Swansea SA2 8PP, U.K. (e-mail: 465186@ swansea.ac.uk; i.sazonov@swansea.ac.uk; p.nithiarasu@swansea.ac.uk).

$\mathrm{X}$. Xie is with the Department of Computer Science, Swansea University, Swansea SA2 8PP, U.K. (e-mail: x.xie@swansea.ac.uk).

Color versions of one or more of the figures in this paper are available online at http://ieeexplore.ieee.org.

Digital Object Identifier 10.1109/TIP.2010.2092434 from volumetric images [1], [2]. However, these techniques are very sensitive to noise and intensity inhomogeneities which exist in real images, and often produce leakages and regions which are not contiguous. Statistical approaches [3], [4] are also used to identify different tissue structures from medical images. It usually involves manual interaction to segment images in order to obtain a sufficiently large set of training samples. Such strategies are often restricted to problems where there is sufficient prior knowledge about the shape or appearance variations of the relevant structures. Also, the use of the same training set for a large number of image scans may lead to biased results that do not take sufficient consideration of the variability within individuals. Atlas based approaches perform segmentation based upon image registration techniques [5], whereby an image can be segmented by finding a transformation that maps a template image to the target image. It is, however, generally difficult for atlas based techniques to accurately extract complex geometries such as those from volumetric medical images due to the variability of anatomical structures.

Another class of segmentation methods partitions an image into different regions based upon energy minimization. These energy based segmentation methods can usually be distinguished as combinatorial methods and variational methods. Graph cuts such as [6]-[8] which are based upon combinatorial optimizations can be used to minimize a cost function defined on a discrete set of variables. In this approach, a graph is composed of vertices representing image pixels or voxels, and edges that connect the vertices. The graph edges are assigned some nonnegative weights or costs, and a cut is a subset of edges that partition the vertices into disjoint sets. The cost function which can consist of boundary and regional information has to be well defined for graph cuts to provide a globally optimal solution. In addition, the discrete representation of the graphs may produce geometric artifacts.

Deformable modeling can be an effective alternative approach. They are usually based upon a variational framework to minimize an energy functional defined on a continuous contour or surface. They have the ability to adapt to complex shape variations and to incorporate priors to regularize segmentation. They have been widely used in applications such as shape extraction [9]-[12] and object tracking [13]-[16]. In these models, curves or surfaces evolve under the influence of both internal and external forces to extract the image object boundaries. Explicit models [17] represent contours and surfaces in their parametric form during deformation. This allows explicit models to track the points on the curves and surfaces across time, and is well suited for real-time applications due to smaller CPU-time requirement. However, explicit models generally have difficulties in dealing with topological changes due to 
the parameterization of the curves and surfaces. McInerney and Terzopoulos [18] designed topology adaptive, explicit deformable models to handle topological changes that often exist in medical image volumes. This explicit model requires a periodic reparameterization mechanism to deal with complex shapes and changes in topology. This technique, however, works well only when the model is required to inflate or deflate everywhere, which considerably limits its applications. Several authors [19]-[21] have also come up with different techniques to handle topological changes. However, these approaches usually involve a set of heuristic algorithms to detect self-intersections and handle splitting and merging of the deforming grid, which can be computationally expensive. Also, these strategies may not work well on structures that consist of complex topologies. Implicit deformable models [9], [10] based upon the theory of curve evolution and the level set method [22], [23] are introduced to address some of these limitations. In these approaches, the evolution of curves and surfaces are represented implicitly as a level set of a higher dimensional scalar function and the deformation of the model is based upon geometric measures such as the unit normal and curvature. Thus, the evolution is independent of the parameterization, and topological changes such as splitting and merging can be handled automatically. In recent years, implicit deformable models have been widely applied in the segmentation of anatomical structures from 3-D medical images [24]-[26].

The design of deformable models often varies in the representation of the object boundary and external force field used. There have been numerous publications on deformable models and improvement of the underlying techniques. These usually take the form of image gradient based approaches, e.g., [10], [27]-[30], region based approaches, e.g., [31] snd [32] and hybrid approaches, e.g., [33] and [34]. Image gradient based techniques have been found useful when there is limited prior knowledge and image gradients are reasonable indications of object boundaries. However, the extension from 2-D to 3-D is not trivial. Conventional image gradient based approaches, such as [10], require careful initialization even in 2-D [32]. Although several improvements have been developed, e.g., [27] and [28], it remains a great challenge for image gradient based models to achieve initialization invariancy and robust convergence. This is especially true when segmenting objects with complex geometries and shapes in 3-D, where delicate manual initialization is even more difficult than in 2-D.

Region based methods [31], [32], [35]-[37] have also been widely applied to image segmentation. The Chan-Vese model [31] which is based upon the Mumford-Shah functional [38] is considered as one of the most popular region based techniques. In this approach, the image is assumed to be composed of regions of approximately piecewise-constant intensities. The Chan-Vese model then extracts the image object based upon the average intensities inside and outside the contour. Although the Chan-Vese model can be used to extract objects with smoothly varying boundaries, it has difficulties dealing with image regions with intensity inhomogeneity. Other region based models such as [36], [37] also assume that image objects consist of distinct regional features, which is often not true for real image dataset due to intensity inhomogeneity and multimodal nature.
In this paper, a novel deformable model with an external force field based upon the relative position and orientation of the deformable model and object boundaries is proposed. A preliminary study on this force interaction has appeared in [39]. The external force field is called the geometric potential force (GPF) field as it is based upon the hypothesized interactions between the relative geometries of the deforming surface and the object boundaries (characterized by image gradients). The evolution of the deformable model is solved using the level set method so as to facilitate topological changes. The proposed external force field can attract the deformable model to object boundaries with arbitrary initialization, and it allows the deformable model to reach highly concave regions which are generally difficult for other methods. The vector force field introduced can be viewed as a generalized version of the magnetic force field described in the recent MAC model [30]. However, the proposed method can be conveniently extended to higher dimensions, unlike for the MAC model, which cannot be directly applied to 3-D image dataset.

The rest of the paper is organized as follows. In Section II, we review several image gradient based methods, particularly some physics-inspired approaches, which are closely related to our method. The proposed method is then described in Section III. The results and comparative studies appear in Section IV. Section V concludes the paper.

\section{Previous Work}

In image gradient based deformable models, it is assumed that object boundaries collocate with image intensity discontinuities. Conventional image gradient based methods, e.g., geometric active contour model [9], [10] and subsequent geodesic models [40], [41] generally have difficulties in dealing with boundary concavities, weak edges, image noise and difficult initializations as they are generally prone to local minima that often appear in real images. Numerous research works have been performed in order to improve the initialization and convergence capabilities of the gradient based approaches.

The gradient vector flow (GVF) and its generalized version GGVF [11], [27] have shown significant improvements over those conventional external force field such as geodesic [40] and have been widely used in deformable models, e.g., [14]. It uses a vector diffusion equation that diffuses the gradient of an edge map to regions distant from the object boundary. The GGVF model may be defined as

$$
\frac{\mathrm{d} C}{\mathrm{~d} t}=\alpha g \kappa \hat{\boldsymbol{n}}+(1-\alpha)(\boldsymbol{v} \cdot \hat{\boldsymbol{n}}) \hat{\boldsymbol{n}}
$$

where $C$ is the deformable contour, $t$ denotes the artificial time component, $g$ is the stopping function, $\kappa$ is the curvature, $\hat{n}$ is the unit normal, and $\alpha$ is a real constant to balance the contribution of the curvature term; $(\boldsymbol{v} \cdot \hat{\boldsymbol{n}}) \hat{\boldsymbol{n}}$ is the flow component normal to the contour. Let $I$ denote an image and $u=|\nabla I|$ be the image gradient magnitude. The diffused GVF field $\boldsymbol{v}(\boldsymbol{x})$ is given as the equilibrium solution to the following PDE:

$$
\frac{\partial \tilde{\boldsymbol{v}}}{\partial t}=w_{1}(|\nabla u|) \nabla^{2} \tilde{\boldsymbol{v}}-w_{2}(|\nabla u|)(\tilde{\boldsymbol{v}}-\nabla u)
$$


where $w_{1}(\cdot)$ and $w_{2}(\cdot)$ are weighting functions which control the amount of diffusion according to the gradient of the edge map. Although the GGVF has been shown to improve the capture range and boundary concavities tracking ability, it still has convergence issues caused by saddle or stationary points in its force field, i.e., when the contour is tangent to the force vector [28], [30], [42].

Recently, there have been several research works on physicsbased deformable models such as [30], [43], [44]. In [43], a charged-particle model (CPM) based upon electrostatics was applied to attract particles toward object boundaries. The authors in [43] hypothesized a set $\Omega$ of freely moving particles with the same positive charge $q$ in an external electrostatic field, generated by fixed negative charges $e_{\boldsymbol{x}}$ proportional to the image gradient $|\nabla I(\boldsymbol{x})|$ at point $\boldsymbol{x}$. The positively charged particles are attracted towards the fixed negative charges under the influence of the external particle-mesh force $\mathbf{F}_{a}$ and repelled by each other by the particle-particle force $\mathbf{F}_{r}$. These forces acting on a moving particle at position $\boldsymbol{x}$ can be computed as the sums

$$
\mathbf{F}_{a}(\boldsymbol{x})=\frac{q}{4 \pi \epsilon} \sum_{\boldsymbol{x}^{\prime} \neq \boldsymbol{x}} e_{\boldsymbol{x}^{\prime}} \frac{\hat{\boldsymbol{r}}_{\boldsymbol{x} \boldsymbol{x}^{\prime}}}{r_{\boldsymbol{x} \boldsymbol{x}^{\prime}}^{2}}, \quad \mathbf{F}_{r}(\boldsymbol{x})=\frac{q^{2}}{4 \pi \epsilon} \sum_{\substack{\boldsymbol{x}^{\prime} \in \Omega \\ \boldsymbol{x}^{\prime} \neq \boldsymbol{x}}} \frac{\hat{\boldsymbol{r}}_{\boldsymbol{x} \boldsymbol{x}^{\prime}}}{r_{\boldsymbol{x} \boldsymbol{x}^{\prime}}^{2}}
$$

where $\epsilon$ is the permittivity, $\hat{\boldsymbol{r}}_{\boldsymbol{x} \boldsymbol{x}^{\prime}}$ is the unit vector pointed from $\boldsymbol{x}$ to $\boldsymbol{x}^{\prime}, r_{\boldsymbol{x} \boldsymbol{x}^{\prime}}=\left|\boldsymbol{x}-\boldsymbol{x}^{\prime}\right|$ is the distance between these two points. The total force acting on a particle is given by $\mathbf{F}=w_{a} \mathbf{F}_{a}+$ $w_{r} \mathbf{F}_{r}+w_{d} \mathbf{F}_{d}$ where $w_{a}, w_{r}$ and $w_{d}$ are weighting parameters for the attraction force $\mathbf{F}_{a}$, repulsion force $\mathbf{F}_{r}$ and damping force $\mathbf{F}_{d}$, respectively. When the particles attain a stable equilibrium state due to the viscous effect of $\mathbf{F}_{d}$, contour reconstruction is required to obtain the object boundary representation. Although this approach can resolve the previously mentioned convergence issues, the fact that particles on weak edges may be attracted to nearby strong edges often causes broken contours to be formed. In addition, the method requires frequent particle insertion and deletion, which is computationally expensive. This makes it impractical in 3-D. In [45], the authors incorporated the particle model [43] into a contour model and showed subsequent improvements on the CPM. However, the dominant external force field is static and its dynamic behavior due to repulsion force can be difficult to predict. Park and Chung in [46] also considered the pixels in the image edge map as static electric charges and used an external force equivalent to $\mathbf{F}_{a}$ in (3) in their parametric model. Similar to [43], their parametric model based upon hypothesized electric field computed from the edge map cannot deal with leakage at weak edges when strong nearby edges are present. Zhu et al. in [47] incorporated tangent direction of the image map to compute a modified version of the electric potential force field. The tangent direction information is obtained by a $90^{\circ}$ rotation of the gradient vectors of the smoothed image, and is coupled with a new parameter that controls the influence of the rotated vectors and the corresponding force field. This enhances the intensity of the hypothesized electric potential along the image edge and allows the active contour to better handle weak edge. However, the performance of the modified external force field in preventing leakage at weak edges is greatly determined by the value of the new parameter, which also affects the noise sensitivity of the active contour. In other words, the noise sensitivity of the modified electric field active contour increases together with its performance in handling weak image edges.

In [30] and [48], Xie and Mirmehdi introduced an external force field that is based upon the hypothesized magnetic force between the active contour and object boundaries. This formulation has been applied directly in the magnetostatic active contour (MAC) to compute the magnetic field and force required to draw the active contour towards object boundaries in 2-D images. This image gradient based method showed significant improvements on convergence issues, e.g., reaching deep concavities, and in handling weak edges and broken boundaries. When applying the analogy directly to deformable modeling, it requires estimation of tangent vectors for the deformable contours, which is convenient in 2-D case, however, not possible in 3-D. Xiang et al. [49] derived an external force for the active contour based upon the elastic interaction (EI) between line defects in solids [50], [51], with its long range attraction force similar to the magnetic force used in MAC [30], [48]. One of the unique properties of MAC and EI is that they take into account the orientation of image gradient vectors in deriving the external force fields, unlike other edge based approaches where only image gradient magnitude are used. We will show later that the MAC model is in fact a special case of our proposed method in 2-D. The new method does not rely on specific initialization as required in the EI model and handles noise interference much better.

Kimmel in [33] also explicitly used image gradient vector directions as an alignment measure in a hybrid approach, coupled with the geodesic active contour and minimal variance criterion suggested by [31]. Given a contour $C$ of length $L$, and in a parametric form $C(s)=\{\boldsymbol{x}(s)\}$ where $s$ is an arc length parameter, the alignment measure used in [33] is given as

$$
E(C)=\int_{0}^{L}|\nabla I[\boldsymbol{x}(s)] \cdot \hat{\boldsymbol{n}}(s)| \mathrm{d} s
$$

where $\hat{\boldsymbol{n}}(s)$ is the unit normal to contour $C$ at $s, \nabla I(\boldsymbol{x})$ is the image gradient at $\boldsymbol{x}$. The alignment measure is used to optimize the orientation of the curve with respect to the image gradients. This measure, together with the gradient-based geodesic measure and the region-based minimal variance criterion is then used to push or pull the contour towards the image boundary. This hybrid approach, however, requires careful tuning of the different parameters associated with various measures in order to efficiently bridge the image gradient and regional information. In addition, only local edge information are used in the alignment measure, while edge information of pixels located away from the contour are not considered in this technique.

\section{PRoposed Method}

Our approach is to define a novel external force field that is based upon hypothesized geometrically induced interactions between the relative geometries of the deformable model and the object boundaries (characterized by image gradients). In other words, the magnitude and direction of the interaction forces are based upon the relative position and orientation between the geometries of the deformable model and image object boundaries, 

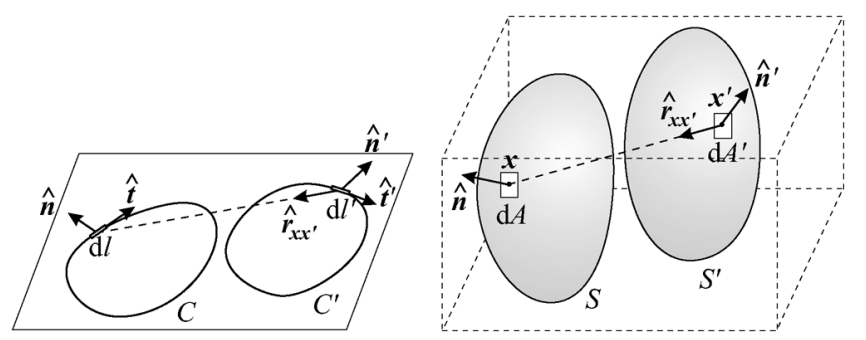

Fig. 1. Relative position and orientation between geometries in 2-D and 3-D.

and hence, it is called the GPF field. The bidirectionality of the new external force field can facilitate arbitrary cross-boundary initialization, which is a very useful feature to have, especially in the segmentation of complex geometries in 3-D. It also improves the performance of the deformable model in handling weak edges. In addition, the proposed external force field is dynamic in nature as it changes according to the relative position and orientation between the evolving deformable model and object boundary.

\section{A. $G P F$}

In order to first deduce the geometric interaction force in 2-D, consider a deformable contour $C$ and an ideal object boundary $C^{\prime}$ in the image plane (see Fig. 1). Let $\mathrm{d} l$ and $\mathrm{d} l^{\prime}$ denote the infinitesimal elements of contour $C$ and object boundary $C^{\prime}$, respectively. In the existing force field based models such as [44], [45], the interaction between $\mathrm{d} l$ and $\mathrm{d} l^{\prime}$ is inversely proportional to the distance separating these two elements and the derived force lies in a straight line between them. They do not take into account the local geometry of the deformable contour $C$ or object boundary $C^{\prime}$. We propose to incorporate the mutual location and orientation of these elements.

Let $\boldsymbol{x}$ and $\boldsymbol{x}^{\prime}$ denote the positions of elements $\mathrm{d} l$ and $\mathrm{d} l^{\prime}$, respectively. Thus, $\boldsymbol{r}_{\boldsymbol{x} \boldsymbol{x}^{\prime}}=\boldsymbol{x}-\boldsymbol{x}^{\prime}$ is their mutual location of those two elements, $r_{\boldsymbol{x} \boldsymbol{x}^{\prime}}=\left|\boldsymbol{x}-\boldsymbol{x}^{\prime}\right|$ is the distance between them, and $\hat{\boldsymbol{r}}_{\boldsymbol{x} \boldsymbol{x}^{\prime}}=\left(\boldsymbol{x}-\boldsymbol{x}^{\prime}\right) / r_{\boldsymbol{x} \boldsymbol{x}^{\prime}}$ is the unit vector pointing $\mathrm{d} l$ from $\mathrm{d} l^{\prime}$. The directions of these elements can be represented by their unit tangent vectors $\hat{\boldsymbol{t}}$ and $\hat{\boldsymbol{t}}^{\prime}$. However, a unique tangent vector is no longer available for infinitesimal surface elements in 3-D. Thus, we use unit outward normal vectors $\hat{\boldsymbol{n}}$ and $\hat{\boldsymbol{n}}^{\prime}$ to characterize the orientations of these elements instead (see Fig. 1). In 2-D, they are simply $90^{\circ}$ rotated tangent vectors.

We are now ready to introduce the hypothesized interaction force $\mathrm{d} \mathbf{F} \mathrm{d} l$ which acts on element $\mathrm{d} l$ by virtue of the hypothesized force field induced by element $\mathrm{d} l^{\prime}$. It is desirable to combine the element orientation vectors and distance vector in deriving the force. We propose a simple but effective combination of these three vectors as $\hat{\boldsymbol{n}}\left(\hat{\boldsymbol{r}}_{\boldsymbol{x} \boldsymbol{x}^{\prime}} \cdot \hat{\boldsymbol{n}}^{\prime}\right)$, unlike CPM [43] as an example where only the distance vector $\hat{\boldsymbol{r}}_{\boldsymbol{x} \boldsymbol{x}^{\prime}}$ is used. The multiplication of contour normal $\hat{\boldsymbol{n}}$ ensures that the force is always imposed in the normal direction so that the deformable model does not suffer from convergence issues (i.e., stationary points, saddle points and extreme boundary concavities), which are often associated with other vector force field based methods such as GVF [27]. The dot product of the object boundary element normal with the distance vector allows the force on the contour in the normal direction to diminish as the contour reaches the object boundary. Similar to other physics-inspired force field, it is also desirable to decay the force interaction with the increase of distance between the elements, i.e., the force is designed proportional to $\hat{\boldsymbol{n}}\left(\hat{\boldsymbol{r}}_{\boldsymbol{x} \boldsymbol{x}^{\prime}} \cdot \hat{\boldsymbol{n}}^{\prime}\right) / r_{\boldsymbol{x} \boldsymbol{x}^{\prime}}^{\lambda}$ where $\lambda>0$. Thus, the contribution of element $\mathrm{d} l^{\prime}$ of object boundary $C^{\prime}$ to the total force acting on $\mathrm{d} l$ in accordance with their distance and mutual orientation can be formulated as

$$
\mathrm{d} \mathbf{F} \mathrm{d} l=\hat{\boldsymbol{n}} \mathrm{d} G \mathrm{~d} l, \quad \mathrm{~d} G=\left(\frac{\hat{\boldsymbol{r}}_{\boldsymbol{x} \boldsymbol{x}^{\prime}}}{r_{\boldsymbol{x} \boldsymbol{x}^{\prime}}^{\lambda}} \cdot \hat{\boldsymbol{n}}^{\prime}\right) \mathrm{d} l^{\prime}
$$

where $\mathbf{F}$ is defined as force per unit length, $\mathrm{d} G$ is the contribution of element $\mathrm{d} l^{\prime}$ of object boundary $C^{\prime}$ into the scalar field $G(\boldsymbol{x})$, which can be considered as an intermediate potential field, and $\lambda$ is a positive constant that affects the magnitude of the interaction force based upon the distance between the elements. In our study, we obtained the best results when $\lambda$ coincides with the dimension of the image data, i.e., $\lambda=2$ in the 2-D case. Furthermore, we show later that when $\lambda$ coincides with data dimension in 2-D, the proposed force interaction has an explicit link to the magnetostatics theory and, thus, the spatial decay of the magnitude of the interaction force is analogous to that of the magnetic field.

As shown in (5), the computation of the new force field only requires unit normal vectors and relative position of the two elements, which is convenient to acquire. Thus, this new force field can be easily extended to higher dimensions, e.g., 3-D. Let $\mathrm{d} A$ belong to the deformable surface $S$ whereas d $A^{\prime}$ belongs to the object boundary $S^{\prime}$ (see Fig. 1). The generalized 3-D version of force $\mathrm{d} \mathbf{F} \mathrm{d} A$ acting between these two area elements can be readily given as

$$
\mathrm{d} \mathbf{F} \mathrm{d} A=\hat{\boldsymbol{n}} \mathrm{d} G \mathrm{~d} A, \quad \mathrm{~d} G=\left(\frac{\hat{\boldsymbol{r}}_{\boldsymbol{x} \boldsymbol{x}^{\prime}}}{r_{\boldsymbol{x} \boldsymbol{x}^{\prime}}^{\lambda}} \cdot \hat{\boldsymbol{n}}^{\prime}\right) \mathrm{d} A^{\prime}
$$

where $\mathbf{F}$ is defined as force per unit area, $G$ is the corresponding 3-D potential field, $\hat{\boldsymbol{n}}$ and $\boldsymbol{n}^{\prime}$ are unit surface normals of the deformable model and object boundary, respectively, and $\lambda=$ 3. Again, the magnitude and direction of the induced force $\mathbf{F}$ is handled intrinsically by the relative position and orientation between the geometries of the deformable model determined by the evolving surface $S$ and object boundary determined by $S^{\prime}$. Since the force is derived geometrically and its interaction is a function of inverse distance, we name it GPF.

\section{B. GPF Deformable Model}

The GPF force in (6) is derived using geometrical information from ideal object boundaries. Next, we extend this to deal with real image data and formulate it in 3-D deformable modelling. In this work, we adopt an edge based approach, that is using image intensity discontinuity to estimate the presence and strength of object boundaries.

Let $I(\boldsymbol{x})$ denote the 3-D image, where $\boldsymbol{x}$ is a voxel location in the image domain. Temporarily, we consider $\boldsymbol{x}$ as a continuously varying point. One may treat this as an interpolation between voxel grid points to obtain a continuous image $I(\boldsymbol{x})$. To compute 
the force acting on $\mathrm{d} A$, we first compute the total potential field for an arbitrary point $\boldsymbol{x}$

$$
G(\boldsymbol{x})=P . V . \oiint_{S^{\prime}} \mathcal{W}\left(\boldsymbol{x}^{\prime}\right)\left(\frac{\hat{\boldsymbol{r}}_{\boldsymbol{x} \boldsymbol{x}^{\prime}}}{r_{\boldsymbol{x} \boldsymbol{x}^{\prime}}^{\lambda}} \cdot \hat{\boldsymbol{n}}^{\prime}\left(\boldsymbol{x}^{\prime}\right)\right) \mathrm{d} A^{\prime} .
$$

where $\mathcal{W}(\cdot)$ is a weighting function that is defined later, and P.V. means 'Principal Value': the contribution of infinitesimal circular vicinity of singular point $\boldsymbol{x}^{\prime}=\boldsymbol{x}$ into the integral is disregarded, which occurs when surfaces $S$ and $S^{\prime}$ intersect.

First, we consider the case, in which $S^{\prime}$ can be defined rigorously on an ideal object $O$, i.e., $S^{\prime}=\partial O$. The object $O$ can be specified by a binary image

$$
\mathcal{I}(\boldsymbol{x})= \begin{cases}\mathcal{I}_{0}, & \boldsymbol{x} \in O \\ 0, & \boldsymbol{x} \notin O\end{cases}
$$

where $\mathcal{I}_{0}$ is a nonzero constant. For such an image, $\nabla \mathcal{I}$ is infinite on $S^{\prime}$ and can be represented through the 3-D Dirac's delta as

$$
\nabla \mathcal{I}(\boldsymbol{x})=\Delta \mathcal{I} \delta\left(\boldsymbol{x}-\boldsymbol{x}^{\prime}\right) \hat{\boldsymbol{n}}^{\prime}\left(\boldsymbol{x}^{\prime}\right)
$$

where $\Delta \mathcal{I}$ is the jump in function $\mathcal{I}(\boldsymbol{x})$ at the boundary of $O$; $\boldsymbol{x}^{\prime} \in S^{\prime}$ and $\hat{\boldsymbol{n}}^{\prime}\left(\boldsymbol{x}^{\prime}\right)$ is the unit normal vector to the surface $S^{\prime}$. Setting $\mathcal{W}$ equal to the jump of $\mathcal{I}$ at the boundary, i.e., $\mathcal{W}=\Delta \mathcal{I}$, we can rewrite (7) as a volume integral

$$
G(\boldsymbol{x})=P . V . \iiint_{\Omega} \mathcal{W}\left(\boldsymbol{x}^{\prime}\right)\left(\frac{\hat{\boldsymbol{r}}_{\boldsymbol{x} \boldsymbol{x}^{\prime \prime}}}{r_{\boldsymbol{x} \boldsymbol{x}^{\prime \prime}}^{\lambda}} \cdot \hat{\boldsymbol{n}}^{\prime}\left(\boldsymbol{x}^{\prime}\right)\right) \delta\left(\boldsymbol{x}^{\prime \prime}-\boldsymbol{x}^{\prime}\right) \mathrm{d} V^{\prime \prime} .
$$

Here, $\boldsymbol{x}^{\prime \prime}$ is the integration variable and $d V^{\prime \prime}$ denotes a volume element. The Dirac's delta is used to obtain the area element from the volume element, i.e., $d A^{\prime} \rightarrow \delta\left(\boldsymbol{x}^{\prime \prime}-\boldsymbol{x}^{\prime}\right) d V^{\prime \prime}$.

Taking into account (9) and $\mathcal{W}=\Delta \mathcal{I}$, we can replace the product $\mathcal{W}\left(\boldsymbol{x}^{\prime}\right) \hat{\boldsymbol{n}}^{\prime}\left(\boldsymbol{x}^{\prime}\right) \delta\left(\boldsymbol{x}^{\prime \prime}-\boldsymbol{x}^{\prime}\right)$ in the integral of (10) by $\nabla \mathcal{I}\left(\boldsymbol{x}^{\prime \prime}\right)$. Thus, (10) can be reformulated as

$$
G(\boldsymbol{x})=P . V \cdot \iiint_{\Omega}\left(\frac{\hat{\boldsymbol{r}}_{\boldsymbol{x} \boldsymbol{x}^{\prime \prime}}}{r_{\boldsymbol{x} \boldsymbol{x}^{\prime \prime}}^{\lambda}} \cdot \nabla \mathcal{I}\left(\boldsymbol{x}^{\prime \prime}\right)\right) \mathrm{d} V^{\prime \prime} .
$$

It is now readily generalizable to real 3-D data.

In real images, $\nabla I$ is a smooth function reaching maximum magnitude in the vicinity of the object boundary. The natural generalization of (11) is to substitute Dirac's delta by this smoothed function analog into (11), i.e., $\mathcal{W}\left(\boldsymbol{x}^{\prime}\right) \hat{\boldsymbol{n}}^{\prime}\left(\boldsymbol{x}^{\prime}\right) \delta\left(\boldsymbol{x}^{\prime \prime}-\boldsymbol{x}^{\prime}\right) \rightarrow \nabla I\left(\boldsymbol{x}^{\prime \prime}\right)$, where $I$ denotes a real image. The geometric potential field in a continuous form can then be formulated as

$$
G(\boldsymbol{x})=P . V \cdot \iiint_{\Omega}\left(\frac{\hat{\boldsymbol{r}}_{\boldsymbol{x} \boldsymbol{x}^{\prime}}}{r_{\boldsymbol{x} \boldsymbol{x}^{\prime}}^{\lambda}} \cdot \nabla I\left(\boldsymbol{x}^{\prime}\right)\right) \mathrm{d} V^{\prime} .
$$

Note, due to the substitution of $\mathcal{W}\left(\boldsymbol{x}^{\prime}\right) \hat{\boldsymbol{n}}^{\prime}\left(\boldsymbol{x}^{\prime}\right) \delta\left(\boldsymbol{x}^{\prime \prime}-\boldsymbol{x}^{\prime}\right)$ by $\nabla I\left(\boldsymbol{x}^{\prime \prime}\right)$, the $\boldsymbol{x}^{\prime}$ defined on the ideal surface $S^{\prime}$ is no longer needed. Hence, the notation is simplified by replacing the integral variable $\boldsymbol{x}^{\prime \prime}$ with $\boldsymbol{x}^{\prime}$. Finally, its discrete form can be written as

$$
G(\boldsymbol{x})=\sum_{\boldsymbol{x}^{\prime} \in \Omega, \boldsymbol{x}^{\prime} \neq \boldsymbol{x}}\left(\frac{\hat{\boldsymbol{r}}_{\boldsymbol{x} \boldsymbol{x}^{\prime}}}{r_{\boldsymbol{x} \boldsymbol{x}^{\prime}}^{\lambda}} \cdot \nabla I\left(\boldsymbol{x}^{\prime}\right)\right) .
$$

This can be considered as a convolution of the image gradient with the vector kernel $\mathbf{K}_{\lambda}(\boldsymbol{x})$

$$
\left\{\begin{array}{l}
\mathbf{K}_{\lambda}(\boldsymbol{x})=P . V \cdot \frac{\hat{\boldsymbol{x}}}{|\boldsymbol{x}|^{\lambda}}=P . V \cdot \frac{\boldsymbol{x}}{|\boldsymbol{x}|^{\lambda+1}} \\
G=\mathbf{K}_{\lambda} * \nabla I \int_{\Omega}\left(\mathbf{K}_{\lambda}\left(\boldsymbol{x}-\boldsymbol{x}^{\prime}\right) \cdot \nabla I\left(\boldsymbol{x}^{\prime}\right)\right) d V^{\prime}
\end{array}\right.
$$

which can be computed efficiently using the fast Fourier transform (FFT). Note that the potential field $G$ is computed as a convolution of two vector functions.

The total force acting on the unit area element of the deformable surface $S$ is, thus, given as $\mathbf{F}=\hat{\boldsymbol{n}} G(\boldsymbol{x})$. where $\hat{\boldsymbol{n}}$ is the outward unit normal of level set surface. Note, an inward normal can also be used, i.e., $\mathbf{F}=-\hat{\boldsymbol{n}} G(\boldsymbol{x})$, which will result in opposite deformable model propagation since the force field is exactly in the opposite direction. An example is given in Fig. 3. Hence, the force can be rewritten in a generalized form

$$
\mathbf{F}=\mathcal{J} \hat{\boldsymbol{n}} G(\boldsymbol{x})
$$

where $\mathcal{J}$ is a constant taking values of \pm 1 . Note this is different from the constant force in the geodesic model, where the force is monotonically expanding or shrinking. The sign convention \pm is merely used to determine whether outward and inward normals of the deformable surface are considered.

The general contrast consistency along the object boundaries, however, is important to the model. Large contrast variation can disrupt the force field, e.g., half of the object appears brighter than background and the other half appears to be darker. However, this does not mean that the entire object has to be brighter or darker than background. Those regions away from object boundary can be continuously varying in intensity (see Fig. 2 as an example).

Once the force field $\mathbf{F}(\boldsymbol{x})$ is derived from the hypothesized interactions based upon the relative geometries of the deformable model and object boundary is determined, the evolution of the deformable model $S(\boldsymbol{x}, t)$ under this GPF field can be given as

$$
\frac{\mathrm{d} S}{\mathrm{~d} t}=(\mathbf{F} \cdot \hat{\boldsymbol{n}}) \hat{\boldsymbol{n}}
$$

Since surface smoothing is usually desirable, the mean curvature flow can be incorporated and the complete GPF deformable model evolution can be formulated as

$$
\frac{\mathrm{d} S}{\mathrm{~d} t}=\alpha g \kappa \hat{\boldsymbol{n}}+(1-\alpha)(\mathbf{F} \cdot \hat{\boldsymbol{n}}) \hat{\boldsymbol{n}}
$$

where $g(\boldsymbol{x})=1 /(1+|\nabla I|)$ is the edge stopping function. Note that in our case, the flow of $\mathbf{F}$ is directed by definition normal to surface $S$, therefore $(\mathbf{F} \cdot \hat{\boldsymbol{n}}) \hat{\boldsymbol{n}}=\mathbf{F}$. Notation $(\mathbf{F} \cdot \hat{\boldsymbol{n}}) \hat{\boldsymbol{n}}$ is inherited from the traditional methods [cf. (1)]. The level set 

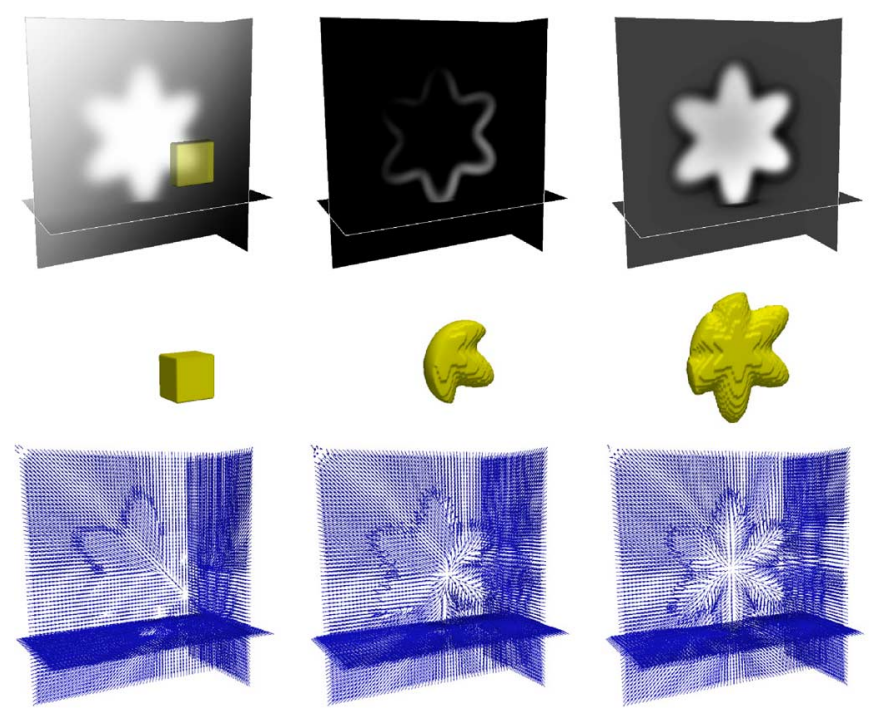

Fig. 2. GPF: first row from left to right-input image and initial deformable model, corresponding edge map and computed geometric potential field, second row-initial and evolving deformable models, and the third row-associated GPF vector field.
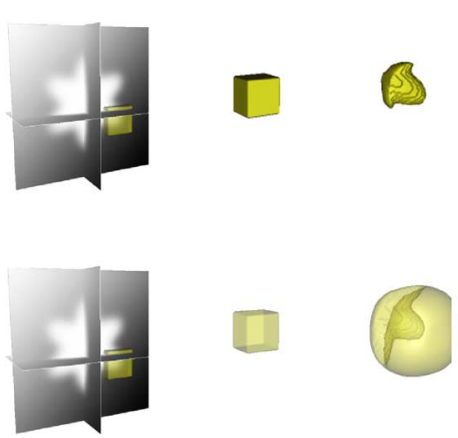

Fig. 3. Direction of propagation of the GPF deformable model. First row $-\mathcal{J}=1$. Second row $-\mathcal{J}=-1$.

representation of the proposed deformable model based upon GPF can then be written as

$$
\frac{\partial \Phi}{\partial t}=\alpha g \kappa|\nabla \Phi|-(1-\alpha)(\mathbf{F} \cdot \nabla \Phi)
$$

where $\Phi(t, \boldsymbol{x})$ is the level set function, such that the deformable surface $S$ is defined as $\Phi(t, x)=0$. Note, the GPF force field is defined on the deformable surface, which is implicitly embedded in the level set function, i.e., the force field computed at the propagating front needs to be extended across the computational domain so that the full level set function can be continuously evolved. Although direct force extension method such as [52] can be used, we can conveniently compute the GPF forces for each level set so that this external force is extended to the entire level set function.

The GPF deformable model differs from conventional edge based models by utilizing edge voxel interactions across the whole image, thus, providing a more global view of the object boundary. The magnitude of the potential field strength at each image location $\boldsymbol{x}$ is based upon the relative position of $\boldsymbol{x}$ with all other voxels in the image. Therefore, voxels at homogeneous regions will also have a nonzero potential field strength. In this way, surfaces which are initialized far away from object boundaries can propagate towards the image edges and converge.

As shown in (6), the dot product $\hat{\boldsymbol{r}}_{\boldsymbol{x} \boldsymbol{x}^{\prime}} \cdot \hat{\boldsymbol{n}}^{\prime}$ can be both positive and negative, depending upon the relative configurations of the geometries between the deformable model and the image boundaries, thus, giving a bidirectional vector force field. This useful bidirectionality facilitates arbitrary cross boundary initializations, as its force vectors point towards the object boundary from both ways. This also allows the model to stabilize the deformable surfaces at weak edges, thus, preventing leakage. The first row of Fig. 2 shows a substantially blurred image with linearly varying intensity, and the corresponding edge map and computed geometric potential field. In addition, as the deformable model evolves, the unit vector $\hat{\boldsymbol{r}}_{\boldsymbol{x} \boldsymbol{x}}$ changes accordingly based upon the relative geometries. This contributes to a vector force field that changes dynamically as the deformable model evolves, as depicted in the second row of Fig. 2. Therefore, the proposed model has much better invariance to its initial position and can deal with complex geometries and extreme boundary concavities.

The physics-based deformable models described in [43]-[47] and reviewed in Section II all use a kernel based function to compute the external force field with kernels being decreasing functions of distance from the origin. They are in effect equivalent to the external force derived in [44] based upon convolving a vector field with the edge map. For example, forces (3) can be represented as a convolution with the same kernel $\mathbf{K}_{\lambda}$ (14) with $\lambda=2$

$$
\mathbf{F}_{a}(\boldsymbol{x})=\frac{q}{4 \pi \epsilon}\left(\mathbf{K}_{\lambda} *|\nabla I|\right), \quad \mathbf{F}_{r}(\boldsymbol{x})=\frac{q^{2}}{4 \pi \epsilon}\left(\mathbf{K}_{\lambda} * 1_{\Omega}\right)
$$

where $1_{\Omega}(\boldsymbol{x})$ is a function equal to 1 when $\boldsymbol{x} \in \Omega$ and 0 otherwise. The repelling force is largely imposed in the tangential direction, which has very limited effect on changing the shape or topology of the deformable model. Thus, it is not necessary in our model. In order to compare with the dominant attraction force $\mathbf{F}_{a}$, we combine (14) and (15) and rewrite the GPF force as

$$
\mathbf{F}_{\mathrm{GPF}}=\mathcal{J} \hat{\boldsymbol{n}}\left(\mathbf{K}_{\lambda} * \nabla I\right) .
$$

It is clear that the GPF force is directed by the normal of the deformable model, i.e., it does not contain the tangential 'parasitic' component in contrast to the $\mathbf{F}_{a}$ force. Moreover, the proposed GPF takes into account edge orientations, as well as edge strength (the convolution in (19) is based upon a convolution of a vector function on a scalar field; whereas in (20) it is carried out on a vector field).

\section{Edge-Preserving Enhancement of Geometric Potential Field Using a Nonlocal Method}

Although the GPF deformable model can reduce its noise sensitivity to a certain extent by modeling gradient vector interactions across the image domain, deformable models based upon image gradients are in general susceptible to heavy noise interference. Note, the GPF force $\mathbf{F}$ is determined by its potential $G$, see (15), and $G$ can be precomputed before evolving the deformable model. Thus, we can improve its performance 
towards image noise by refining the potential $G$. Here, we enhance it using nonlocal methods [53], [54], so as to increase its robustness even in the presence of a large amount of image noise. One main advantage in choosing nonlocal methods over local diffusion or averaging methods [55], [56] is the ability to preserve fine structures. Moreover, the potential field has zero or close to zero magnitude in the middle of object boundaries, with positive and negative potential values immediately on either side. Using local methods to carry out smoothing, there is a risk of canceling out on object boundaries, which is not desirable. In nonlocal methods [53], [54], similarity is not measured based upon a single pixel/voxel value but is measured based upon the neighborhood of the pixel/voxel. In particular, nonlocal methods not only compares the intensity value in a single pixel/voxel, but also the geometrical configuration in its neighborhood. This gives a more robust performance than local smoothing filters. In a standard nonlocal denoising algorithm [53], an image pixel/voxel is restored using the weighted average of all the pixels/voxels in the image. This algorithm, however, cannot be applied directly to the denoising of the geometrically induced potential field $G$. This is because edge information in the geometric potential field is represented differently from that of the original image. For instance, the gray level values of the original image on the same side of object edges can be quite similar, thus, giving a large weight for similarity measure. On the other hand, the difference in magnitudes of the geometric potential field at these regions can, however, be very large, giving a small weight for similarity measure, causing similar structures to be considered as different. Therefore, instead of comparing voxel neighborhood from the geometric potential field to refine itself, we measure voxel similarity from the original image and use the computed similarity weights to refine the geometric potential.

Given the noisy image $I(\boldsymbol{x})$ and its corresponding geometric potential field $G(\boldsymbol{x})$, this nonlocal smoothing of the geometric potential field is carried out by computing a weighted average at each voxel position $\boldsymbol{x}$ according to

$$
G^{\prime}(\boldsymbol{x})=\sum_{\boldsymbol{x}^{\prime} \in \Omega_{\boldsymbol{x}}, \boldsymbol{x}^{\prime} \neq \boldsymbol{x}} w\left(\boldsymbol{x}, \boldsymbol{x}^{\prime}\right) G(\boldsymbol{x})
$$

where $\Omega_{\boldsymbol{x}}$ is a search window centered around $\boldsymbol{x}$. Following the approach in Buades et al. [53], we measure the similarity between two square (2-D) or cube (3-D) regions centered at location $\boldsymbol{x}$ and $\boldsymbol{x}^{\prime}$, and determine the similarity weight from the image $I(\boldsymbol{x})$ as

$$
w\left(\boldsymbol{x}, \boldsymbol{x}^{\prime}\right)=\frac{1}{Z(\boldsymbol{x})} e^{-\frac{1}{h^{2}}\left(\sum_{\delta \in \boldsymbol{N}} G_{\sigma}(\delta)\left(I(\boldsymbol{x}+\delta)-I\left(\boldsymbol{x}^{\prime}+\delta\right)\right)^{2}\right)^{2}}
$$

where $G_{\sigma}($.$) is a Gaussian kernel with standard deviation \sigma, \boldsymbol{N}$ denotes the region containing the pixels/voxels location $\delta, h$ is the parameter that controls the amount of filtering, and $Z(\boldsymbol{x})$ is a normalization constant given by $Z(\boldsymbol{x})=\sum_{\boldsymbol{x}^{\prime} \in \Omega_{\boldsymbol{x}}} w\left(\boldsymbol{x}, \boldsymbol{x}^{\prime}\right)$. The force acting due to the enhanced geometrical potential field on the deformable surface $S$ described in (15) can then be given as

$$
\begin{aligned}
\mathrm{d} \mathbf{F}(\boldsymbol{x}) & =\mathcal{J} \mathrm{d} A_{1} \hat{\boldsymbol{n}}(\boldsymbol{x}) G^{\prime}(\boldsymbol{x}) \\
& =\mathcal{J} \mathrm{d} A_{1} \hat{\boldsymbol{n}}(\boldsymbol{x}) \sum_{\boldsymbol{x}^{\prime} \in \Omega_{\boldsymbol{x}}, \boldsymbol{x}^{\prime} \neq \boldsymbol{x}} w\left(\boldsymbol{x}, \boldsymbol{x}^{\prime}\right) G(\boldsymbol{x}) .
\end{aligned}
$$

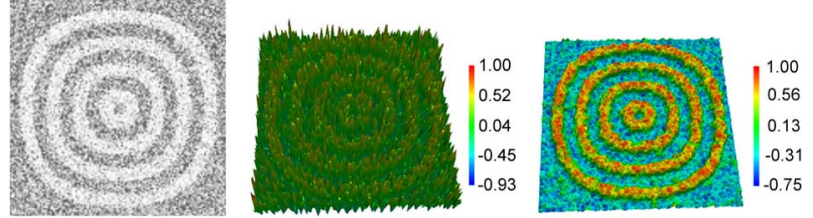

Fig. 4. Edge-preserving nonlocal enhancement of geometric potential field: from left-example image with noise, geometric potential field $G(\boldsymbol{x})$ and the enhanced $G^{\prime}(\boldsymbol{x})$. The potential fields shown as 3-D surface plots.
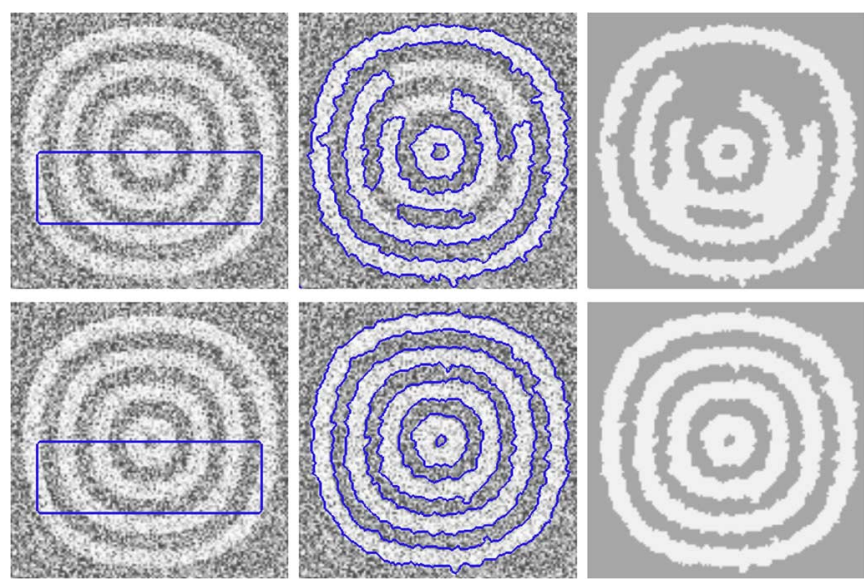

Fig. 5. Comparative results of concentric rings segmentation from noisy image. First row: EI model. Second row: proposed GPF model.

By comparing regional similarity instead of single pixel/ voxel similarity from the noisy image, a more reliable geometric potential field is achieved. Moreover, oscillations at flat or homogeneous regions are readily smoothed and edge information at object boundaries are enhanced in the denoised geometric potential field. Fig. 4 shows a 2-D image with 70\% added Gaussian noise, the computed geometric potential field $G(\boldsymbol{x})$ and the enhanced $G^{\prime}(\boldsymbol{x})$ using nonlocal filtering. It is evident that this nonlocal method can efficiently remove noise interference and preserves edge information in the geometric potential field. This greatly enhances the performance of the deformable model in handling image noise. The second row of Fig. 5 provides the segmentation result on this substantially noise corrupted image using the enhanced geometric potential field.

\section{Relationship With MAC and Comparison With EI Models}

In the MAC models [30], [48], the external force field is based upon the hypothesized magnetic force between the active contour $C$ and object edge $C^{\prime}$. Interaction between two elements $\mathrm{d} l$ and $\mathrm{d} l^{\prime}$ of contours $C$ and $C^{\prime}$, respectively, is described in accordance with the Biot-Savart law

$$
\mathrm{d} \mathbf{F} \mathrm{d} l=\Upsilon \mathrm{d} l(\hat{\boldsymbol{t}} \times \mathrm{d} \mathbf{B}), \quad \mathrm{d} \mathbf{B}=\frac{\mu_{0}}{4 \pi} \frac{\Upsilon^{\prime} \mathrm{d} l^{\prime}}{r_{\boldsymbol{x}^{\prime} \boldsymbol{x}}^{2}}\left(\hat{\boldsymbol{t}}^{\prime} \times \hat{\boldsymbol{r}}_{\boldsymbol{x}^{\prime} \boldsymbol{x}}\right)
$$

where $\Upsilon$ and $\Upsilon^{\prime}$ are electric currents in contours $C$ and $C^{\prime}$, respectively; $\hat{\boldsymbol{t}}$ and $\hat{\boldsymbol{t}}^{\prime}$ are unit tangent vectors to elements $\mathrm{d} l$ and $\mathrm{d} l^{\prime}$, respectively; $r_{\boldsymbol{x}^{\prime} \boldsymbol{x}}$ is the distance between $\mathrm{d} l$ and $\mathrm{d} l^{\prime}, \hat{\boldsymbol{r}}_{\boldsymbol{x}^{\prime} \boldsymbol{x}}$ is the unit vector pointing from $\mathrm{d} l^{\prime}$ to $\mathrm{d} l ; \mu_{0}$ is the permeability constant. The current directions represented by the tangent vectors $\hat{t}$ and $\hat{\boldsymbol{t}}^{\prime}$ have to be known in advance before computing 
the magnetic field and force. To deal with this requirement, the authors in [30] and [48] compute the direction of the imposed currents for the active contour and object boundary by rotating the respective gradient vectors in a clockwise or anti-clockwise manner such that a current loop is formed on both the active contour and object boundary.

As shown in [30] and [48], the MAC method has many advantages when dealing with objects of complicated topology, with noise, and weak edges. However, it is difficult to extend MAC to handle 3-D images directly as it is not apparent how the hypothesized current direction is to be estimated and set on a 3-D object. Here we show that the MAC model is a particular case of the proposed method in 2-D.

Notice that tangent vectors $\hat{\boldsymbol{t}}$ and $\hat{\boldsymbol{t}}^{\prime}$ can be represented as a cross-product of normals $\hat{\boldsymbol{n}}$ and $\hat{\boldsymbol{n}}^{\prime}$ and the unit vector $\hat{\boldsymbol{z}}=$ $(0,0,1)$ normal to the image plane: $\hat{\boldsymbol{t}}=\hat{\boldsymbol{z}} \times \hat{\boldsymbol{n}}$ and $\hat{\boldsymbol{t}}^{\prime}=\hat{\boldsymbol{z}} \times \hat{\boldsymbol{n}}^{\prime}$ (see Fig. 1). Then applying Lagrange's formula for the vector triple product we obtain

$$
\begin{aligned}
(\hat{\boldsymbol{z}} \times \hat{\boldsymbol{n}}) \times \mathrm{d} \mathbf{B} & =-\hat{\boldsymbol{z}}(\hat{\boldsymbol{n}} \cdot \mathrm{d} \mathbf{B})+\hat{\boldsymbol{n}}(\hat{\boldsymbol{z}} \cdot \mathrm{d} \mathbf{B}) \\
& =\hat{\boldsymbol{n}}(\hat{\boldsymbol{z}} \cdot \mathrm{d} \mathbf{B}), \\
\left(\hat{\boldsymbol{z}} \times \hat{\boldsymbol{n}}^{\prime}\right) \times \hat{\boldsymbol{r}}_{\boldsymbol{x}^{\prime} \boldsymbol{x}} & =-\hat{\boldsymbol{z}}\left(\hat{\boldsymbol{n}}^{\prime} \cdot \hat{\boldsymbol{r}}_{\boldsymbol{x}^{\prime} \boldsymbol{x}}\right)+\hat{\boldsymbol{n}}^{\prime}\left(\hat{\boldsymbol{z}} \cdot \hat{\boldsymbol{r}}_{\boldsymbol{x}^{\prime} \boldsymbol{x}}\right) \\
& =-\hat{\boldsymbol{z}}\left(\hat{\boldsymbol{n}}^{\prime} \cdot \hat{\boldsymbol{r}}_{\boldsymbol{x}^{\prime} \boldsymbol{x}}\right) .
\end{aligned}
$$

Now we can rewrite (24) in the following form (ignoring constants)

$$
\mathrm{d} \mathbf{F} \mathrm{d} l=\Upsilon \mathrm{d} l \hat{\boldsymbol{n}}(\hat{\boldsymbol{z}} \cdot \mathrm{d} \mathbf{B}), \quad \mathrm{d} \mathbf{B}=-\frac{\Upsilon^{\prime} \mathrm{d} l^{\prime}}{r_{\boldsymbol{x}^{\prime} \boldsymbol{x}}^{2}} \hat{\boldsymbol{z}}\left(\hat{\boldsymbol{r}}_{\boldsymbol{x}^{\prime} \boldsymbol{x}} \cdot \hat{\boldsymbol{n}}^{\prime}\right)
$$

Note that the magnetic field in the 2-D model has only a vertical component: $\mathrm{d} \mathbf{B}=(0,0, \mathrm{~d} B)=\mathrm{d} B \hat{\boldsymbol{z}}$ where $\mathrm{d} B=(\hat{\boldsymbol{z}} \cdot \mathrm{d} \mathbf{B})$. Hence, by setting $\mathrm{d} B=\mathrm{d} G, \Upsilon=1$ and $\Upsilon^{\prime}=1$ and also taking into account that $\hat{\boldsymbol{r}}_{\boldsymbol{x}^{\prime} \boldsymbol{x}}=-\hat{\boldsymbol{r}}_{\boldsymbol{x} \boldsymbol{x}^{\prime}}$ and $r_{\boldsymbol{x}^{\prime} \boldsymbol{x}}=r_{\boldsymbol{x} \boldsymbol{x}^{\prime}}$, it will lead us to the 2-D GPF model as in (5) with $\lambda=2$. If we set $\Upsilon=\mathcal{J}$ and $\Upsilon^{\prime}=|\nabla I|$, this will show that the image gradient based MAC model is equivalent to the proposed image gradient weighted GPF in 2-D as given in (13) and (15), again with $\lambda=2$. Thus, we can consider the proposed method as a generalization of the MAC method [30] to higher dimensions or, in other words, the MAC method is a special case of GPF in 2-D.

The force field used in the EI model [49], [57] is defined based upon the EI between line defects and can be given as

$$
\tilde{\boldsymbol{v}}=P . V . \oiint_{\Omega} \frac{\hat{\boldsymbol{r}}_{\boldsymbol{x} \boldsymbol{x}^{\prime}}}{r_{\boldsymbol{x} \boldsymbol{x}^{\prime}}^{2}} \cdot \nabla\left(G_{\sigma} * I+\beta \mathcal{H}_{\epsilon}(\Phi)\right) \mathrm{d} A
$$

where $\boldsymbol{x}$ and $\boldsymbol{x}^{\prime}$ are points on the moving contour and on the object boundary, respectively, $G_{\sigma}$ is a Gaussian kernel with standard deviation $\sigma, \beta$ is an adjustable coefficient, and $\mathcal{H}_{\epsilon}$ is a smoothed Heaviside function. The EI force $\tilde{\boldsymbol{v}}$ in (28) consists of an image based interaction force and a contour based interaction force. The image based interaction is given by the dot product $\left(\hat{\boldsymbol{r}}_{\boldsymbol{x} \boldsymbol{x}^{\prime}} / r_{\boldsymbol{x} \boldsymbol{x}^{\prime}}^{2}\right) \cdot \nabla\left(G_{\sigma^{*}} * I\right)$ and takes a similar form to the proposed geometric interactions. The term $\left(\hat{\boldsymbol{r}}_{\boldsymbol{x} \boldsymbol{x}^{\prime}} / r_{\boldsymbol{x} \boldsymbol{x}^{\prime}}^{2}\right) \cdot \nabla\left(\beta \mathcal{H}_{\epsilon}(\Phi)\right)$ represents the contour based interaction within the moving contour.

The EI model initializes the contour using the zero-crossing of the image based interaction force, which sets the initial contour near the object boundary. This also generates many false contours caused by spurious edges. It is assumed that the force generated by the noise is relatively small as compared to the interaction force at the object boundary. The EI model then uses the contour based interaction force within the evolving contour to overcome the interaction between the noise and the contour. The parameter $\beta$, thus, needs to be sufficiently large in order to shrink the spurious edges caused by the noise. This initialization strategy, however, is only suitable when the user intends to extract all the objects in the image. It is therefore not appropriate to use this initialization technique to extract a single object from multiple objects that exist in the image. For example, in order to segment the aorta and the femoral bone from the medical images shown in Figs. 12 and 15, respectively, the user should be able to place the initial contour or surface inside, outside or across the feature of interest. However, with a user-defined initialization, it is difficult to select the parameter $\beta$, since a small $\beta$ may not be able to handle the image noise, and a large $\beta$ may overwhelm weak edges and the deformable model may not be able to propagate through boundary concavities as an example. It is also noted that the curvature flow used in the formulation of the EI model is not coupled with an edge stopping function which generally exists in geometric deformable models. The weighting for the curvature term in the EI model can be set to a large value, so as to have significant influence on the contour evolution and to overcome the noise. However, without the edge stopping function, a large curvature force may easily overwhelm weak edges or even shrink the correct contour at the object boundary. Moreover, the computation of the contour based interaction force is required at every time step during the contour evolution. Although this can be accelerated by using FFT, it is still requires a significant amount of computational effort, especially when dealing with 3-D images.

The proposed method, however, does not rely on any specific initialization strategy. The deformable model can be placed to localize single or multiple regions of interest with arbitrary cross boundary initializations. Compared to EI, it is more effective in removing noise interference and more efficient in evolving the deformable model. Fig. 5 gives such an example. A heavily noise corrupted image containing concentric rings with a cross boundary initialization is used for the test. The EI model was found struggling to find the right balance in overcoming image noise and propagating the contour further. The proposed method evolves much more efficiently and achieved a much better result.

\section{E. Implementation and Setting of Parameters}

The image object boundary description used in the computation of $G(x)$ can be acquired from the derivatives of the image intensity using central difference, or standard edge detection methods such as the Sobel filter as used here. Some erroneous edges with very small magnitude were removed, i.e., 5\%-10\% of the maximum magnitude. The curvature flow term used in the level set formulation is calculated using central difference, and the force term is computed based upon an upwind scheme. The Narrow Band approach described in [23] is used to update the level set function so as to reduce the computational cost of embedding the contour or surface in a higher dimensional function.

As mentioned in Section III-B, $\mathcal{J}= \pm 1$ can be used to determine the direction of propagation of the deformable model. In 
particular, the deformable model will extract relatively brighter voxels from an image region when $\mathcal{J}=1$, and extract the darker voxels when $\mathcal{J}=-1$ as shown in Fig. 3. $\mathcal{J}=1$ is used for all other examples in the paper. However, as shown earlier in Fig. 3, a cross boundary initialization often results in the same result.

The enhancement of the geometric potential field using nonlocal method can be computationally expensive if we use a search window $\Omega_{x}$ of size similar to that of the image. For computational purposes, a search window of $21 \times 21$ pixels for 2 -D images as suggested in [53] and $21 \times 21 \times 21$ voxels for 3 -D images are generally sufficient for most images, and a square or cube similarity neighborhood region $\boldsymbol{N}$ of length 7 for 2-D and 3-D images is usually large enough for noise robustness and small enough to preserve fine structures and details [53]. In addition, the nonlocal filtering can be accelerated using efficient algorithms such as the combination of summed square image scheme and FFT used in [58], and the vectorization and parallelization strategy described in [54]. The parameter $h$ in (22) controls the amount of filtering which depends upon the amount of noise present in the image. Note that refining of the geometric potential field is only necessary when the noise presence is significant. The proposed GPF model can handle considerable amount of image noise as the geometric potential field is computed using edge pixel or voxel interactions across the whole image.

\section{RESUlTS AND DISCUSSION}

In this section, we present experimental results on both synthetic and real 3-D image data. The algorithms are implemented in $\mathrm{C}++$, and the computations are performed on an Intel Core 2 Duo $3.00 \mathrm{GHz}$ processor with 4 GB RAM. The comparative analysis is performed using several classical and state-of-the-art methods which consists of image gradient based and region based methods. In particular, the geodesic model is included as a representative of conventional local edge fitting based method which is based upon monotonically expanding or shrinking force. The various vector field based models, such as [43]-[47], have very similar convergence and initialization dependence behavior to the GVF or GGVF, since their dominant external forces are static as discussed earlier. Thus, the GGVF model is used as a representative of vector force field based approaches. In addition, the Chan-Vese model with its region based formulation, and the more recent EI model which uses a dynamic vector force, are also included in the analysis.

\section{A. Multiple Objects}

The first column in Fig. 6 shows the shape extraction results for the six-ellipsoids problem. Given an arbitrary initialization across all the ellipsoids, only GPF could accurately recover the shapes. The geodesic model, given the same initialization configuration, simply expanded outwards and reached the image borders. This is due to the fact that the geodesic model cannot handle cross-boundary initialization as the constant pressure term can only monotonically shrink or expand the contour. Although the bidirectionality of the GGVF model enables it to handle cross-boundary initialization, the saddle and stationary

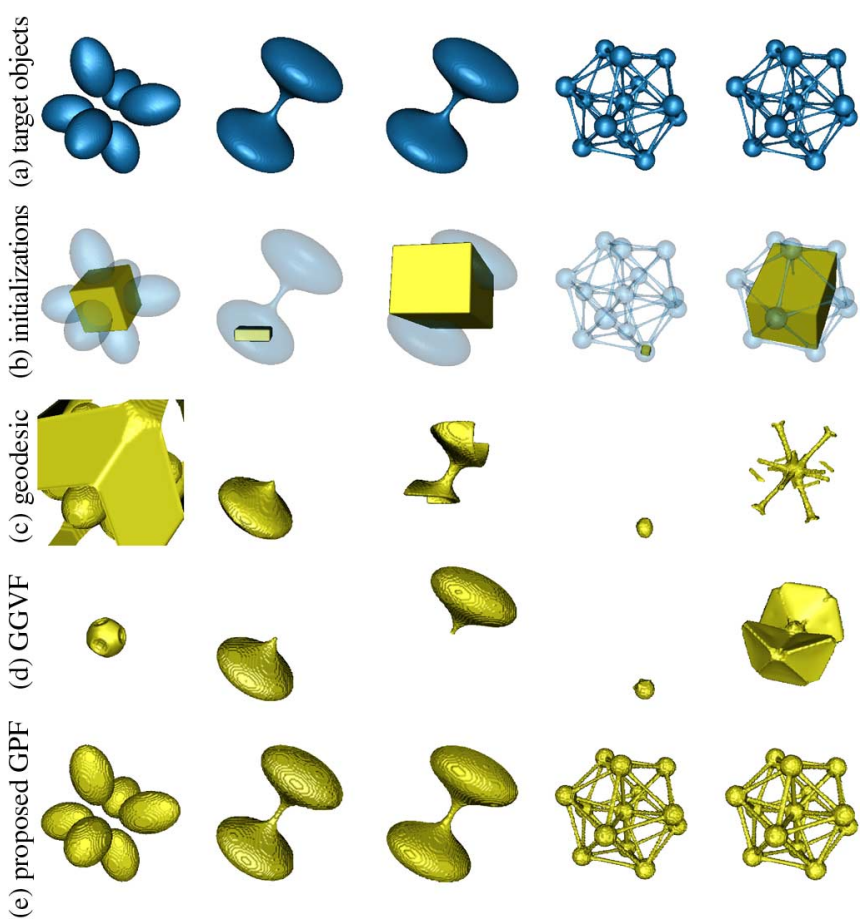

Fig. 6. Shape recovery from synthetic images. (a) Isosurfaces of various shapes to be recovered from synthetic images $(128 \times 128 \times 128)$. (b) Initial deformable models (yellow) with input shapes (blue, semitransparent). (c) Recovered shape using geodesic. (d) GGVF. (e) Proposed GPF.

points in this example prevented GGVF from extracting the ellipsoids.

\section{B. Convergence to Boundary Concavities}

Next, we compare the ability of the deformable models to deal with highly concave boundaries. As shown in the second and third columns in Fig. 6, the geometrical object to be recovered consists of two flattened ellipsoids connected by a narrowing tube with a constriction in the middle. With the deformable models initialized inside one of the ellipsoid, only GPF could propagate through the narrowing tube to accurately extract the shape. Also, with a more arbitrary cross-boundary initialization, GPF was the only successful model to extract the exact shape. The other two methods could neither handle the arbitrary initialization nor propagate through deep concavities. Note that the bottleneck between the two ellipsoids is extremely narrow, which makes it particularly difficult for geodesic model to propagate through without stepping through the object boundary due to the large expansion force.

\section{Handling Complex Geometries and Topologies}

The fourth and fifth columns in Fig. 6 compares the shape extraction results on a complex geometry with different initialization configurations. When the initial surface is placed inside one of the sphere of the molecular structure, GPF is the only model that managed to extract the geometry successfully. The other two models were not able to propagate through the long narrow tubes connecting the spheres. With a cross-boundary initialization, the geodesic model only recovered part of the geometry with a negative pressure force, while GGVF converges to 
TABLE I

Comparative Results on the SEGMENTATION OF VARIOUS SHAPES FROM SYNTHETIC IMAGES: FOREGROUND (FG), BACKGROUND (BG) AND OVERALL ACCURACY MEASURED IN \%

\begin{tabular}{l||lccr}
\hline \hline \multicolumn{1}{|||}{$\begin{array}{l}\text { Six-ellipsoids } \\
\text { cross initialization }\end{array}$} & FG (\%) & 93.9 & 0.20 & 99.9 \\
& BG (\%) & 0.15 & 99.3 & 100 \\
& Overall (\%) & 47.0 & 49.7 & $\mathbf{9 9 . 9}$ \\
\hline Ellipsoids with & FG (\%) & 41.7 & 48.5 & 99.5 \\
narrowing tube & BG (\%) & 100 & 100 & 100 \\
inside initialization & Overall (\%) & 70.8 & 74.2 & $\mathbf{9 9 . 8}$ \\
\hline Ellipsoids with & FG (\%) & 3.79 & 48.5 & 99.5 \\
narrowing tube & BG (\%) & 99.8 & 100 & 100 \\
cross initialization & Overall (\%) & 51.8 & 74.2 & $\mathbf{9 9 . 8}$ \\
\hline Molecular & FG (\%) & 4.73 & 5.51 & 99.9 \\
inside initialization & BG (\%) & 100 & 100 & 99.7 \\
& Overall (\%) & 52.4 & 52.8 & $\mathbf{9 9 . 8}$ \\
\hline Molecular & FG (\%) & 12.0 & 10.6 & 100 \\
cross initialization & BG (\%) & 99.8 & 95.6 & 99.7 \\
\multicolumn{1}{|l}{ FG Average (\%) } & Overall (\%) & 55.9 & 53.1 & $\mathbf{9 9 . 8}$ \\
\hline \hline BG Average (\%) & & 31.2 & 22.7 & $\mathbf{9 9 . 8}$ \\
Overall Average (\%) & & 80.0 & 99.0 & $\mathbf{9 9 . 9}$ \\
\hline
\end{tabular}

the wrong shape due to the saddle and stationary regions in the complex geometry.

The previous examples demonstrate the superior performance of the GPF deformable model in resolving deep concavities and handling complex geometries and topologies. This is mainly due to the dynamic nature of the vector force field. In addition, we show that the bidirectionality of the new force field gives GPF the flexibility to deal with arbitrary cross-initializations.

Table I presents the comparative results for the recovery of various shapes shown in Fig. 6. The foreground (FG) and background (BG) accuracy of the extracted shapes were measured as the percentages of true foreground and background voxels which were actually segmented as foreground and background, respectively. Note that each of the synthetic images used in the examples shown in Fig. 6 has an image size of $128^{3}$, and contains a relatively large number of background voxels compared to the foreground voxels, e.g., the percentages of foreground and background voxels in the six-ellipsoids images are $7.60 \%$ and $92.4 \%$, respectively. Therefore, we use a normalized overall accuracy given as the average of FG and BG to measure the accuracy of correctly extracted voxels from the image, so as to prevent measurement bias towards the large number of background voxels. The geodesic model is shown to give an average FG and BG accuracy of $31.2 \%$ and $80.0 \%$, respectively, and an average overall accuracy of $55.6 \%$. The GGVF model provides an average FG accuracy of $22.7 \%$, an average BG accuracy of $99.0 \%$ and an overall average of $60.8 \%$. The GPF model clearly outperforms the others with a significantly higher FG and BG average accuracy of $99.8 \%$ and $99.9 \%$, respectively, and an overall average of $99.8 \%$.

\section{Recovery of Weak Object Boundaries}

Fig. 7 shows a harmonic shape with smoothly varying image intensity and substantially diffused or blurred boundary segment. The geodesic model with the initial surface placed inside the object as shown in the first row, leaks through the weak edge. The GGVF model, due to the bidirectionality of its force field, can converge to the weak edge with careful initialization.

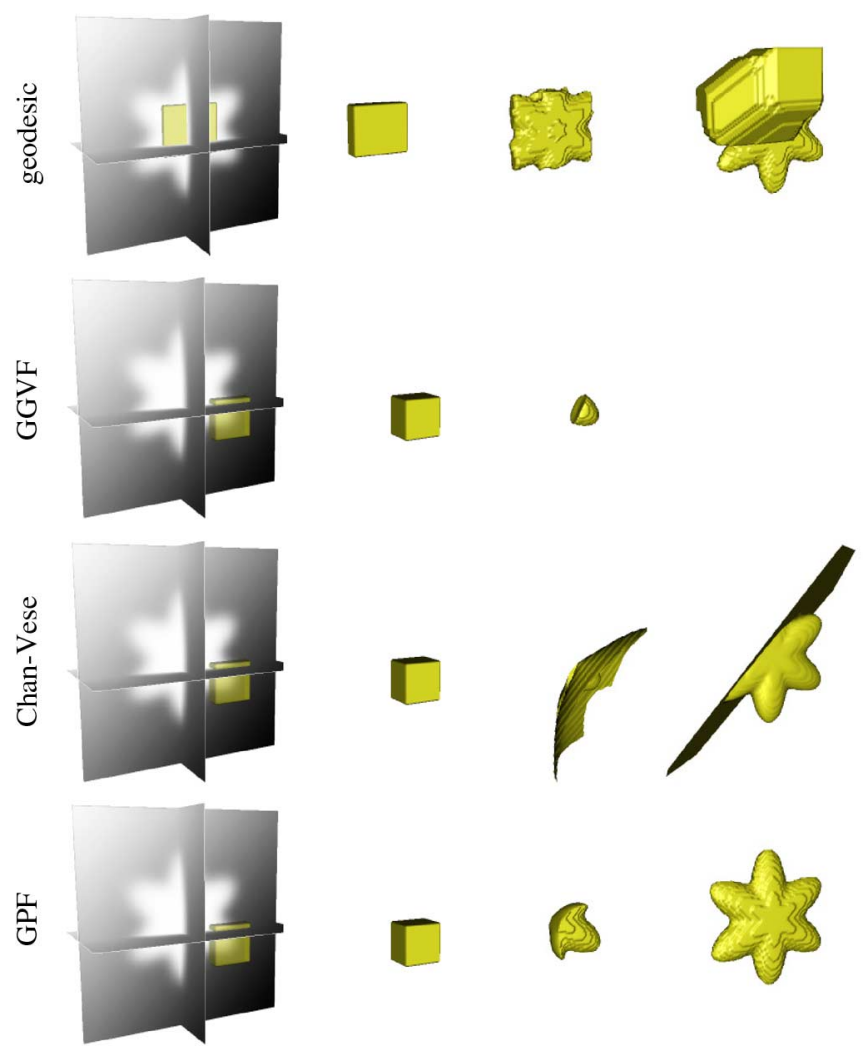

Fig. 7. Shape recovery from weak edges. First row: geodesic. Second row: GGVF. Third row: Chan-Vese. Fourth row: proposed GPF.

However, a more arbitrary cross-boundary initialization causes the deformable model to collapse as shown in the second row. The Chan-Vese model has difficulties propagating across the image due to intensity inhomogeneity as shown in the third row of Fig. 7. GPF, on the other hand, can efficiently localize the object despite the weak boundary, intensity inhomogeneity, and cross-boundary initialization, as shown in the last row.

\section{E. Initialization Invariancy}

The bidirectional and dynamic force field gives the GPF model a high invariance to various arbitrary initializations, therefore providing a high consistency in segmentation results. For example in Fig. 6, the geodesic and GGVF models both give very different segmentation results when the models are initialized differently. Conversely, the GPF model converged to the correct object boundaries given different initializations. More arbitrary initializations are used in the complex geometries in Fig. 8 to further illustrate the initialization invariancy of the GPF model. In the first and third rows of Fig. 8, the initial surface is placed across one end of the double helix shape and molecular structure, respectively. In the second and fourth rows of Fig. 8, a uniformly distributed initialization is used to segment the complex geometries. No discernable difference is found in the results produced through different initializations.

\section{F. Robustness to Image Noise}

As the geometric potential field is computed using edge voxel interactions across the whole image, it provides a more global view of the object boundary representation. This makes the GPF 

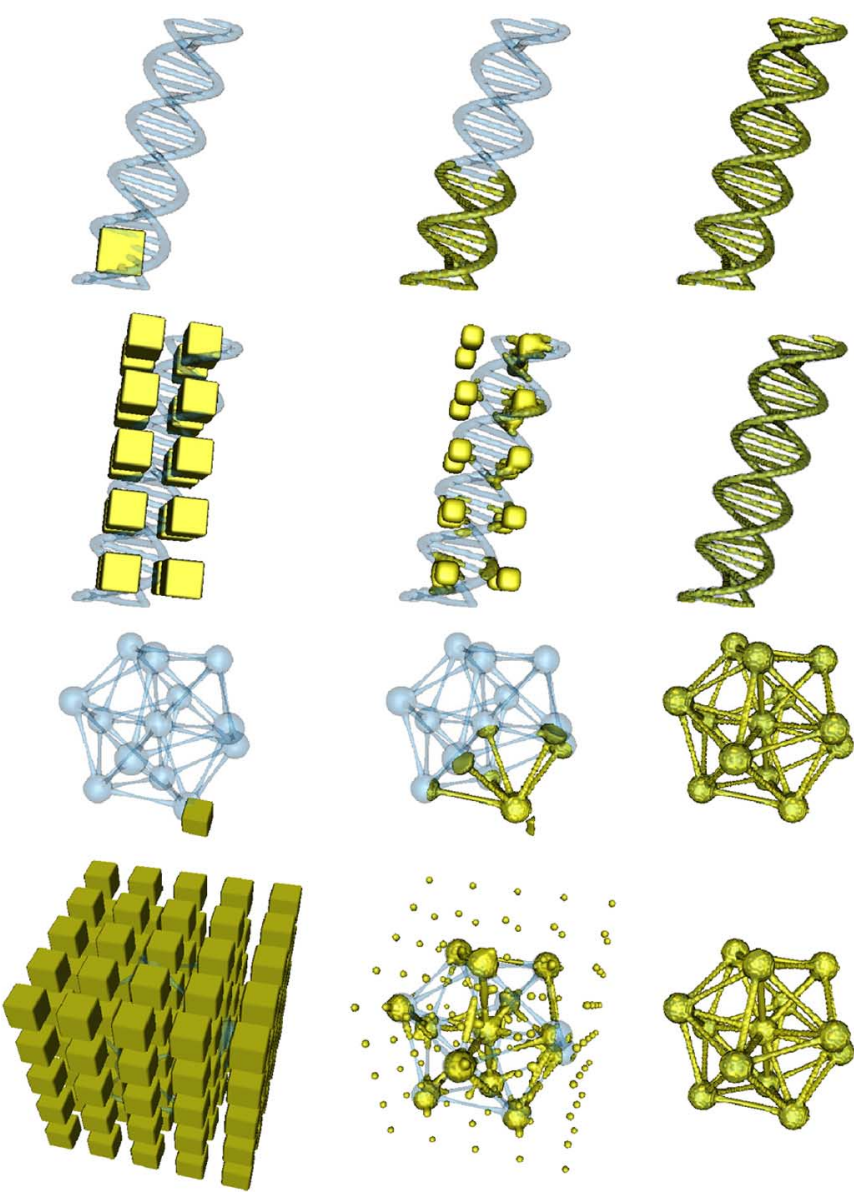

Fig. 8. Arbitrary initialization using GPF (yellow—deformable surfaces, semitransparent blue-isosurfaces from synthetic images): First and second rows: double helix shape segmentation using different initializations. Third and fourth rows: molecular shape segmentation using different initializations.

model more robust to image noise compared to other models which use only local image gradient information. In addition, the edge-preserving denoising of the geometric potential field described in Section III-C can significantly reduce its sensitivity to noise.

Fig. 9 shows the robustness of the GPF model on image noise, using the geometric potential field computed directly from the noisy image (i.e., without denoising). It accurately extracted the shape from the noisy image. Note the substantial amount of noise made it difficult for the marching cubes based algorithm to render the target object (cf. Fig. 6). Fig. 10 illustrates the increase in noise robustness of the GPF model, using the nonlocal denoising algorithm on the geometric potential field. In this particular example, a considerable amount of nonuniform noise was further added to the noisy image from Fig. 9. As depicted in Fig. 10, the substantial amount of noise added produces some localized noise concentration or artifacts. Although the EI model with its contour based interaction force can shrink the spurious edges caused by the large amount of noise, it prevented the deformable model from propagating across the image object. A smaller weight for the contour based force will result in numerous erroneous regions. The deformable model using the potential field computed directly from the noisy image managed to extract the foreground ob-

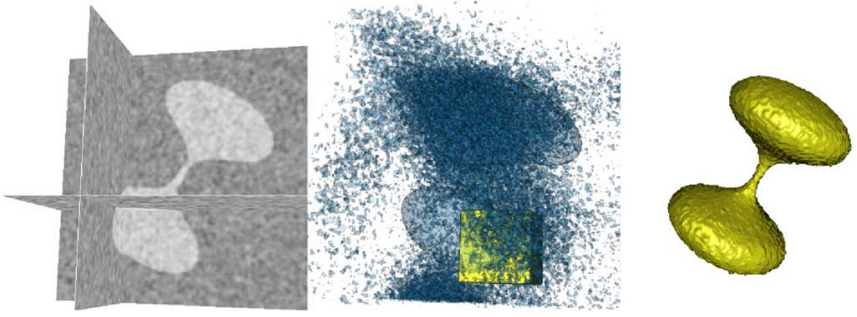

Fig. 9. Shape recovery from noisy image-from left: noisy image, isosurface with initialization (yellow), and recovered shape using the GPF model.
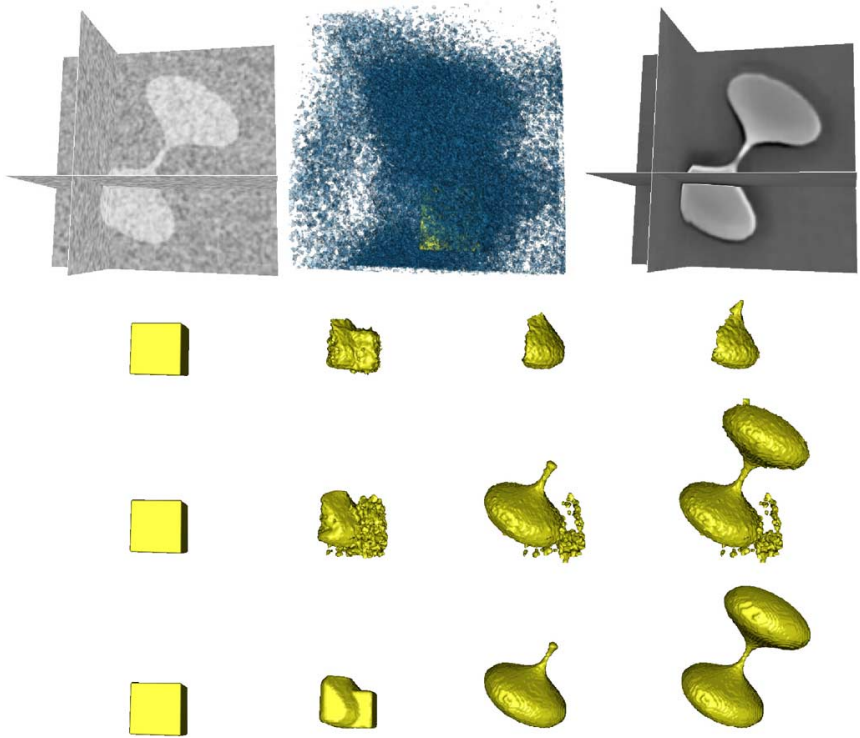

Fig. 10. Shape recovery using edge-preserving nonlocal enhancement of geometric potential field - first row: noisy image $(128 \times 128 \times 128)$, isosurface with initialization (yellow), and denoised geometric potential field using nonlocal method; second row: evolving deformable surfaces using the EI model (CPU-time, $109590 \mathrm{~s}$ ); third row: evolving deformable surfaces using the GPF model with original geometric potential field from noisy image (CPU-time, $1846 \mathrm{~s}$ ); fourth row: evolving deformable surfaces using the GPF model with denoised geometric potential field using nonlocal method (CPU-time, $932 \mathrm{~s}$ ).

ject, but it also produced some erroneous edges. On the other hand, an enhanced geometric potential field using the nonlocal method greatly increases the accuracy of the deformable model in segmenting the object from the noisy image. Note that no filtering such as Gaussian or anisotropic smoothing was applied. The EI model took $109590 \mathrm{~s}$ to converge in this example due to the high computation cost of its contour based interaction force at each iteration, while the GPF model achieve a much faster convergence using $1846 \mathrm{~s}$ and $932 \mathrm{~s}$ with the original and denoised geometric potential field, respectively. Fig. 11 compares the profiles of the geometric potential field before and after nonlocal denoising. The noise fluctuation shown in the original profile of geometric potential field can introduce some false edges to the segmentation. However, the nonlocal denoising method described previously can remove the noise interference and enhance the true object boundary information.

\section{G. Segmentation of Real Images}

Real images often contain complex geometries and topologies, image noise and weak edges. Here, we show some compar- 

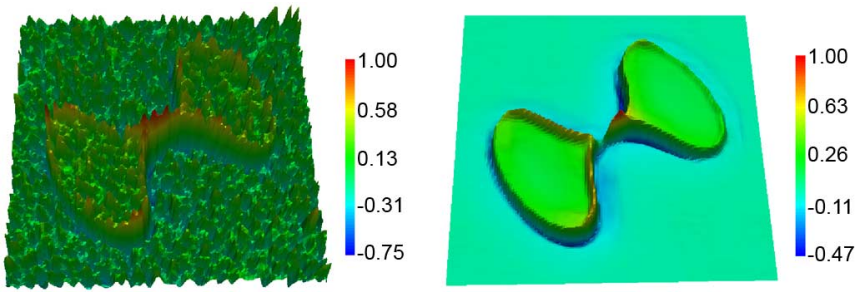

Fig. 11. Edge-preserving nonlocal denoising of geometric potential field-left: geometric potential field (normalized) of noisy image before nonlocal denoising; right: geometric potential field (normalized) of noisy image with nonlocal denoising.
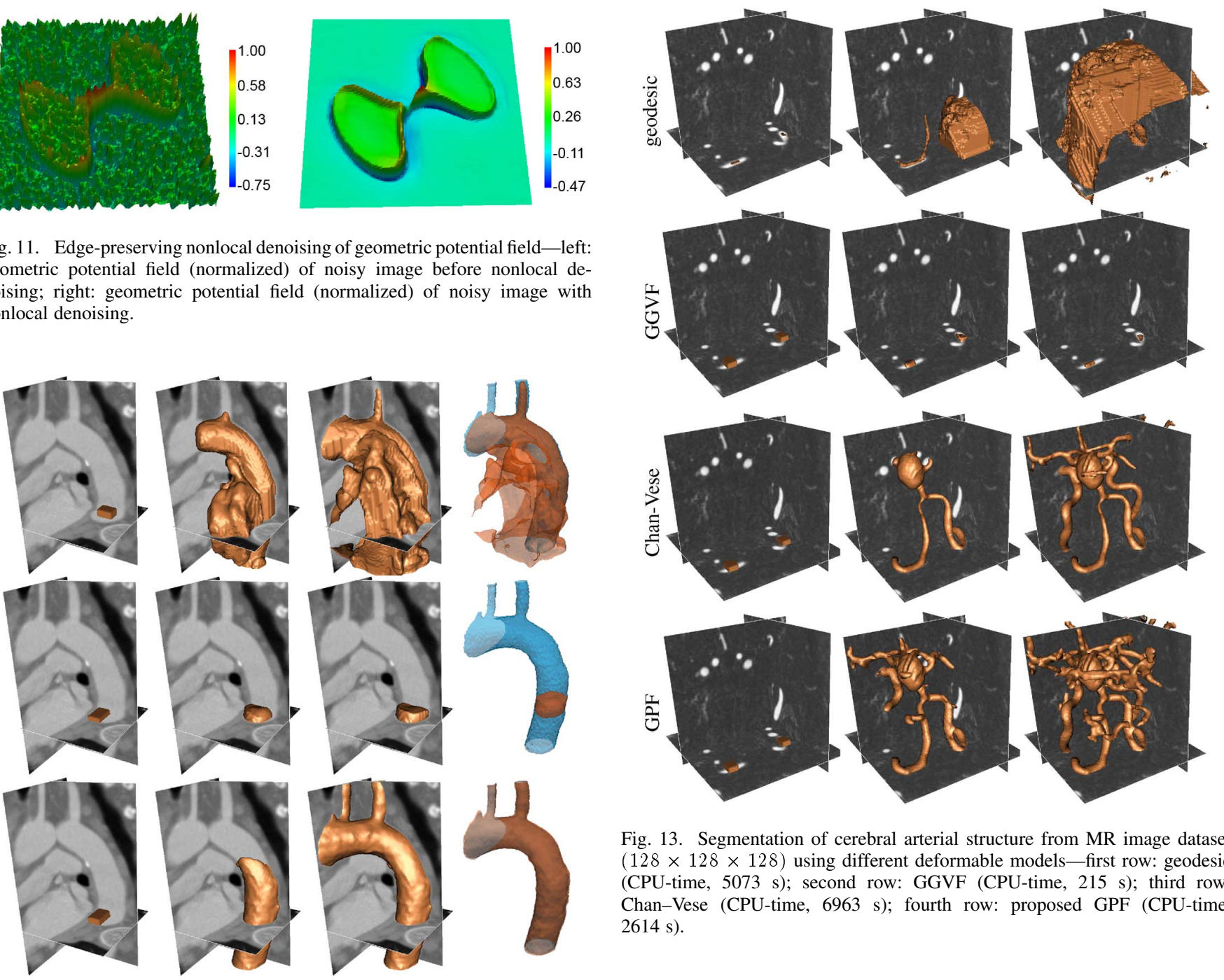

Fig. 13. Segmentation of cerebral arterial structure from MR image dataset $(128 \times 128 \times 128)$ using different deformable models-first row: geodesic (CPU-time, $5073 \mathrm{~s}$ ); second row: GGVF (CPU-time, 215 s); third row: Chan-Vese (CPU-time, 6963 s); fourth row: proposed GPF (CPU-time, 2614 s).

Fig. 12. Segmentation of human aorta from CT image dataset $(64 \times 64 \times 128)$ using different deformable models-first row: geodesic (CPU-time, $2416 \mathrm{~s}$ ); second row: GGVF (CPU-time, 104 s); third row: proposed GPF (CPU-time, $1388 \mathrm{~s}$ ). The last column shows the comparison of segmented geometries (orange) with manual segmentation (blue).

ative results on the segmentation of 3-D medical data. Fig. 12 shows the segmentation of a human aorta from computed tomography (CT) images. In this example, a simple global image thresholding was applied to remove the dark regions which represent air cavity in the image. This preprocessing was applied so as to minimize the interference in segmenting the aorta. As the geodesic model cannot handle cross-boundary initialization, the initial surface is placed within the image structure as shown in the first row. The constant pressure term of the geodesic model easily overwhelms the weak edges and causes some leakage which expands towards the image boundary. A more arbitrary initialization is used for GGVF and GPF as shown in the second and third rows, respectively. The GGVF model did not propagate through the main aortic structure and collapsed to nearby edges, while the GPF model accurately converged to the geometry of the aorta. Note that in order for GGVF to converge properly, the initial surface needs to be placed very close to the aorta boundaries, which is impractical in 3-D. The last column compares the segmentation results with the geometry reconstructed from manual segmentation (shown in blue). It is found that although the geodesic model acquired a FG accuracy of $97.4 \%$ for this example, the corresponding BG accuracy acquired is less than $50 \%$ due to the leakage through weak edges. The GGVF model had difficulties propagating through the long narrow structure and only provided a FG accuracy of 7.3\%, while the GPF model acquired a FG, BG, and overall accuracy of $99.8 \%, 98.7 \%$, and $99.2 \%$, respectively.

Fig. 13 shows comparative results on the segmentation of cerebral arterial structure from magnetic resonance (MR) imaging. Two initial surfaces are placed inside the object of interest for the geodesic model, and across the object boundaries for GGVF, Chan-Vese and GPF. The geodesic model cannot propagate through the narrow tubular structures, and leaks out at weak object boundaries during the evolution. The GGVF model collapsed to the nearby object edges due to the saddle or stationary points inside the narrow image structures. In contrast, the Chan-Vese and GPF models are able to propagate through the long tubular structures to extract the cerebral arterial geometry. The EI model can be initialized near the boundary of the cerebral structure using the zero-crossing of the image based interaction force, and the image noise can be subsequently removed with its contour based interaction force 

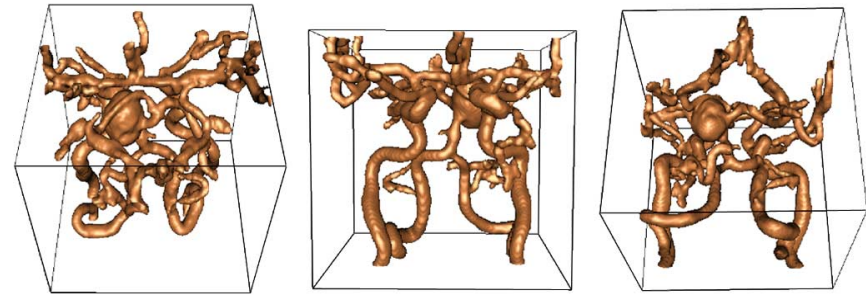

Fig. 14. Different views of the segmented cerebral arterial model using the GPF deformable model.
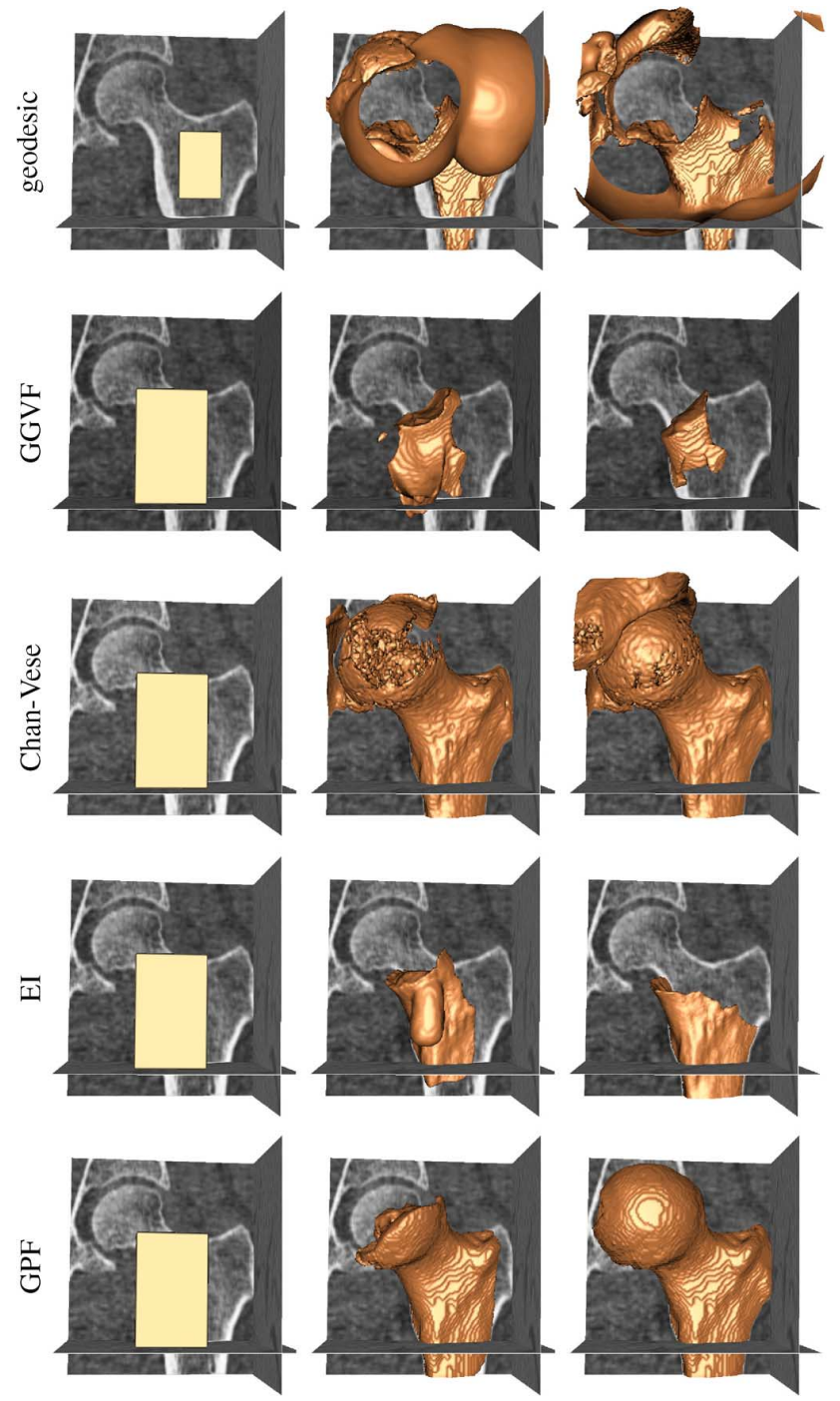

Fig. 15. Segmentation of femur from CT image dataset $(128 \times 128 \times 128)$ using different deformable models-first row: geodesic (CPU-time, $4176 \mathrm{~s}$ ); second row: GGVF (CPU-time, $1250 \mathrm{~s}$ ); third row: Chan-Vese (CPU-time, $10060 \mathrm{~s}$ ); fourth row: EI (CPU-time, $163520 \mathrm{~s}$ ); fifth row: proposed GPF (CPU-time, $2423 \mathrm{~s}$ ).

to extract the geometry. However, this specific initialization strategy cannot be applied to segment the aorta and the femoral bone from the medical images with multiple objects shown in Fig. 12 and Fig. 15, respectively. Various views of the segmented cerebral arterial model using GPF is depicted in Fig. 14. Fig. 15 presents another example whereby a femur is segmented from CT images using the different methods. In

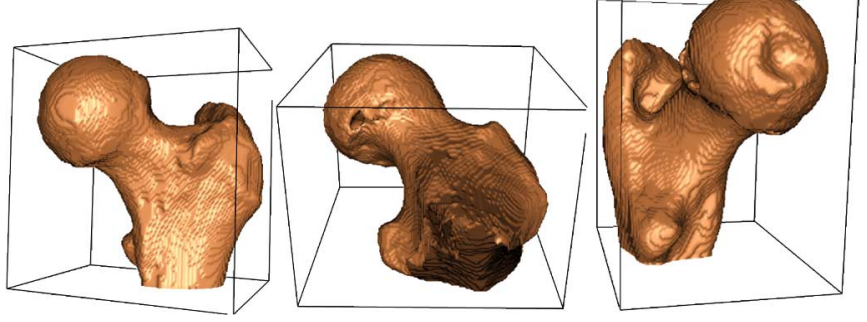

Fig. 16. Different views of the segmented femoral model using the GPF deformable model.

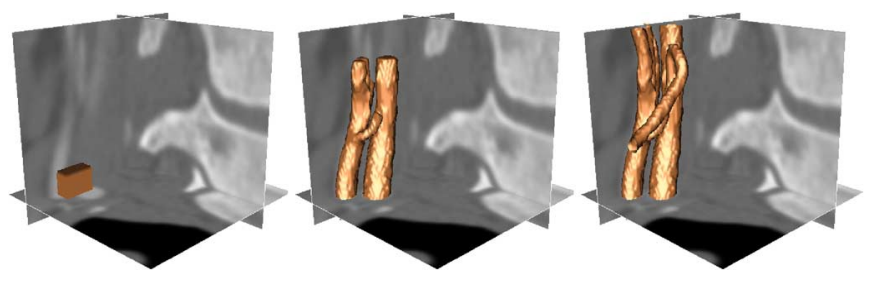

Fig. 17. Segmentation of multiple branches of the carotid from CT image dataset $(120 \times 120 \times 60)$ using GPF (CPU-time, $2581 \mathrm{~s})$.

this example, the geodesic model and the Chan-Vese model leaked due to the weak image edges and varying intensities, respectively, while the GGVF and EI models had difficulties in propagating across the image object. The GPF model, however, can effectively extract the image object despite the image noise, weak edges and inhomogeneous intensities. Fig. 16 shows the various views of the femoral model segmented using the GPF model. Fig. 17 depicts the segmentation of multiple branches of the carotid using GPF. The previous examples have shown that the GPF deformable model can efficiently segment thin and complex structures, and can handle inhomogeneity in image intensities, noises and weak edges, which are often present in real images. The improvements achieved by the proposed method, as demonstrated extensively in various examples, are significant and consistent.

\section{CONCLUSion}

We have presented a novel deformable model that uses an external force field known as the GPF, which is computed based upon the relative geometrical configurations between the deformable model and image object. The proposed method utilizes pixel or voxel interactions across the whole image, which effectively provides a global representation of the image object. The derived geometric potential field is, thus, more informative and exhibits spatial and structural characteristics of image objects which are more coherent than image cues that are based solely on local edge or regional information. This makes the new model more robust towards image noise and weak object edges. The relativity between geometries gives the proposed deformable model its distinctive bidirectionality, which facilitates the handling of arbitrary cross-boundary initializations. In addition, the new framework is equipped with a dynamic vector force field that adapts appropriately to the relative position and orientation between the geometries as the deformable model evolves. This allows the proposed deformable 
model to seamlessly handle complex geometries and topologies efficiently. The dynamic vector force can easily attract the deformable model into highly concave regions, and propagate the contour or surface through long thin structures. The enhancement of the geometric potential field based upon regional similarity measure, can effectively remove noise interference, and yet preserve object edge information and fine details. We provided several comparative examples against existing edge based and region based techniques using various geometries and topologies from both synthetic and real images. The comparative study clearly showed that the proposed method achieved significant improvements in convergence capability and initialization flexibility and outperformed many competing methods. The straightforward generalization of the proposed model to higher dimensions allows the framework to be applied on N-dimensional images, and opens up to a wide range of potential applications.

\section{REFERENCES}

[1] C. M. Smith, J. Smith, S. K. Williams, J. J. Rodriguez, and J. B. Hoying, "Automatic thresholding of three-dimensional microvascular structures from confocal microscopy images," J. Microsc., vol. 225, no. 3, pp. 244-257, 2007.

[2] J. Wu, F. Ye, J. Ma, X. Sun, J. Xu, and Z. Cui, "The segmentation and visualization of human organs based on adaptive region growing method," in Proc. Int. Conf. Comp. Inf. Tech., 2008, pp. 439-443.

[3] S. Ruan, C. Jaggi, J. Xue, J. Fadili, and D. Bloyet, "Brain tissue classification of magnetic resonance images using partial volume modeling," IEEE Trans. Pattern Anal. Mach. Intell., vol. 19, no. 12, pp. 1179-1187, Dec. 2000.

[4] J. Hao and M. Li, "A supervised bayesian method for cerebrovascular segmentation," WSEAS Trans. Signal Process., vol. 3, no. 12, pp. 487-495, 2007.

[5] J. B. Maintz and M. A. Viergever, "A survey of medical image registration," Med. Image Anal., vol. 2, no. 1, pp. 1-36, 1998.

[6] Y. Boykov and G. Funka-Lea, "Graph cuts and efficient n-d image segmentation," Int. J. Comput. Vis., vol. 70, no. 2, pp. 109-131, 2006.

[7] J. Shi and J. Malik, "Normalized cuts and image segmentation," IEEE Trans. Pattern Anal. Mach. Intell., vol. 22, no. 8, pp. 888-905, Aug. 2000.

[8] P. F. Felzenszwalb and D. P. Huttenlocher, "Efficient graph-based image segmentation," Int. J. Comput. Vis., vol. 59, no. 2, pp. 167-181, 2004.

[9] V. Caselles, F. Catte, T. Coll, and F. Dibos, "A geometric model for active contours," Numer. Math., vol. 66, pp. 1-31, 1993.

[10] R. Malladi, J. A. Sethian, and B. C. Vemuri, "Shape modelling with front propagation: A level set approach," IEEE Trans. Pattern Anal. Mach. Intell., vol. 17, no. 2, pp. 158-175, Feb. 1995.

[11] C. Xu and J. L. Prince, "Generalized gradient vector flow external forces for active contours," Signal Process., vol. 71, no. 2, pp. 131-139, 1998.

[12] R. Whitaker, "Modeling deformable surfaces with level sets," Comput. Graph. App., vol. 24, no. 5, pp. 6-9, 2004.

[13] N. Paragois and R. Deriche, "Geodesic active contours and level sets for the detection and tracking of moving objects," IEEE Trans. Pattern Anal. Mach. Intell., vol. 22, no. 3, pp. 226-280, Mar. 2000.

[14] N. Ray and S. Acton, "Motion gradient vector flow: An external force for tracking rolling leukocytes with shape and size constrained active contours," IEEE Trans. Med. Imag., vol. 23, no. 12, pp. 1466-1478, Dec. 2004.

[15] M. Niethammer and A. Tannenbaum, "Dynamic geodesic snakes for visual tracking," in Proc. IEEE Conf. Comput. Vis. Pattern Recognit., 2004, pp. 660-667.

[16] Y. Rathi, N. Vaswani, A. Tannenbaum, and A. Yezzi, "Particle filtering for geometric active contours with application to tracking moving and deforminb objects," in Proc. IEEE Conf. Comput. Vis. Pattern Recognit., 2005, pp. 2-9.

[17] M. Kass, A. Witkin, and T. Terzopoulos, "Snakes: Active contour models," Int. J. Comput. Vis., vol. 1, no. 4, pp. 321-331, 1987.
[18] T. McInerney and D. Terzopoulos, "Topology adaptive deformable surfaces for medical image volume segmentation," IEEE Trans. Med. Imag., vol. 18, no. 10, pp. 840-850, Oct. 1999.

[19] H. Delingette and J. Montagnat, "Shape and topology constraints on parametric active contours," J. Comput. Vis. Image Understand., vol. 83, no. 2, pp. 140-171, 2001.

[20] J. Bredno, T. M. Lehmann, and K. Spitzer, "A general discrete model in two, three, and four dimensions for topology-adaptive multichannel segmentation," IEEE Trans. Pattern Anal. Mach. Intell., vol. 25, no. 5, pp. 550-563, May 2003.

[21] J.-O. Lauchaud and B. Taton, "Deformable model with a complexity independent from image resolution," CVIU, vol. 99, no. 3, pp. 453-475, 2005.

[22] S. Osher and J. Sethian, "Fronts propagating with curvature-dependent speed: Algorithms based on the hamilton-jacobi formulation," J. Comp. Phys., vol. 79, pp. 12-49, 1988.

[23] J. A. Sethian, Level Set Methods and Fast Marching Methods: Evolving Interfaces in Computational Geometry, Fluid Mechanics, Computer Vision, and Material Science. Cambridge, U.K.: Cambridge Univ. Press, 1999.

[24] C. Baillard and C. Barillot, "Robust 3-D segmentation of anatomical structures with level sets," in Proc. MICCAI, 2000, pp. 236-245.

[25] M. Law and A. Chung, "A deformable surface model for vascular segmentation," in Proc. MICCAI, 2009, pp. 59-67.

[26] M. Holtzman-Gazit, R. Kimmel, N. Peled, and D. Goldsher, "Segmentation of thin structures in volumetric medical images," IEEE Trans. Image Process., vol. 15, no. 2, pp. 354-363, Feb. 2006.

[27] C. Xu and J. L. Prince, "Snakes, shapes, and gradient vector flow," IEEE Trans. Image Process., vol. 7, no. 3, pp. 359-369, Mar. 1998.

[28] N. Paragios, O. Mellina-Gottardo, and V. Ramesh, "Gradient vector flow geometric active contours," IEEE Trans. Pattern Anal. Mach. Intell., vol. 26, no. 3, pp. 402-407, Mar. 2004.

[29] C. Li, J. Liu, and M. Fox, "Segmentation of edge preserving gradient vector flow: An approach toward automatically initializing and splitting of snakes," in Proc. IEEE Conf. Comput. Vis. Pattern Recognit., 2005, pp. $162-167$.

[30] X. Xie and M. Mirmehdi, "MAC: Magnetostatic active contour model," IEEE Trans. Pattern Anal. Mach. Intell., vol. 30, no. 4, pp. 632-647, Apr. 2008.

[31] T. Chan and L. Vese, "Active contours without edges," IEEE Trans. Image Process., vol. 10, no. 2, pp. 266-277, Feb. 2001.

[32] D. Cremers, M. Rousson, and R. Deriche, "A review of statistical approaches to level set segmentation: Integrating color, texture, motion and shape," Int. J. Comput. Vis., vol. 72, no. 2, pp. 195-215, 2007.

[33] R. Kimmel, Geometric Level Set Methods in Imaging, Vision, and Graphics. Berlin, Germany: Springer-Verlag, 2003, ch. Fast edge integration, pp. 59-77.

[34] X. Xie and M. Mirmehdi, "RAGS: Region-aided geometric snake," IEEE Trans. Image Process., vol. 13, no. 5, pp. 640-652, May 2004.

[35] L. Vese and T. Chan, "A multiphase level set framework for image segmentation using the mumford and shah model," Int. J. Comput. Vis., vol. 50, no. 3, pp. 271-293, 2002.

[36] N. Paragios and R. Deriche, "Geodesic active regions and level set methods for supervised texture segmentation," Int. J. Comput. Vis., vol. 46, no. 3, pp. 223-247, 2002.

[37] J. Kim, J. W. Fisher, A. Yezzi, M. Cetin, and W. A. S. , "A nonparametric statistical method for image segmentation using information theory and curve evolution," IEEE Trans. Med. Imag., vol. 14, no. 10, pp. 1486-1502, Oct. 2005.

[38] D. Mumford and J. Shah, "Optimal approximations by piecewise smooth functions and associated variational problems," Commun. Pure Appl. Math., vol. 42, no. 5, pp. 577-685, 1989.

[39] S. Y. Yeo, X. Xie, I. Sazonov, and P. Nithiarasu, "Geometric potential force for the deformable model," in Proc. BMVC, 2009, pp. 1-11.

[40] V. Caselles, R. Kimmel, and G. Sapiro, "Geodesic active contour," Int. J. Comput. Vis., vol. 22, no. 1, pp. 61-79, 1997.

[41] K. Siddiqi, Y. Lauzière, A. Tannenbaum, and S. Zucker, "Area and length minimizing flows for shape segmentation," IEEE Trans. Image Process., vol. 7, no. 3, pp. 433-443, Mar. 1998.

[42] D. Gil and P. Radeva, "Curvature vector flow to assure convergent deformable models for shape modelling," in Proc. EMMCVPR, 2003, pp. 357-372.

[43] A. Jalba, M. Wilkinson, and J. Roerdink, "CPM: A deformable model for shape recovery and segmentation based on charged particles," IEEE Trans. Pattern Anal. Mach. Intell., vol. 26, no. 10, pp. 1320-1335, Oct. 2004. 
[44] B. Li and S. Acton, "Active contour external force using vector field convolution for image segmentation," IEEE Trans. Image Process., vol. 16, no. 8, pp. 2096-2106, Aug. 2007.

[45] R. Yang, M. Mirmehdi, and X. Xie, "A charged active contour based on electrostatics," in Proc. ACIVS, 2006, pp. 173-184.

[46] H. K. Park and M. J. Chung, "External force of snake: Virtual electric field," Electron. Lett., vol. 38, no. 24, pp. 1500-1502, 2002.

[47] G. Zhu, S. Zhang, Q. Zeng, and C. Wang, "Anisotropic virtual electric field for active contours," Patter Recognit. Lett., vol. 29, no. 11, pp. 1659-1666, 2008.

[48] X. Xie and M. Mirmehdi, "Magnetostatic field for the active contour model: A study in convergence," in Proc. BMVC, 2006, pp. 127-136.

[49] Y. Xiang, A. Chung, and J. Ye, "A new active contour method based on elastic interaction," in Proc. IEEE Conf. Comput. Vis. Pattern. Recognit., 2005, pp. 452-457.

[50] J. P. Hirth and J. Lothe, Theory of Dislocations. Hoboken, NJ: Wiley, 1982.

[51] E. M. L. L. D. Landau, Statistical Physics. New York: Pergamon, 1980.

[52] D. Adalsteinsson and J. Sethian, "The fast construction of extension velocities in level set methods," J. Comp. Phys., vol. 148, pp. 2-22, 1998.

[53] A. Buades, B. Coll, and J. Morel, "A non-local algorithm for image denoising," in Proc. IEEE Conf. Comput. Vis. Pattern Recognit., 2005, pp. 60-65.

[54] J. Darbon, A. Cunha, T. Chan, S. Osher, and G. Jensen, "Fast nonlocal filtering applied to electron cryomicroscopy," in Proc. IEEE Int. Symp. Biomed. Imag.: From Nano to Macro, 2008, pp. 1331-1334.

[55] P. Perona and J. Malik, "Scale space and edge detection using anisotropic diffusion," IEEE Trans. Pattern Anal. Mach. Intell., vol. 12, no. 7, pp. 629-639, Jul. 1990.

[56] C. Tomasi and R. Manduchi, "Bilateral filtering for gray and color images," in Proc. IEEE Int. Conf. Comput. Vis., 1998, pp. 839-846.

[57] Y. Xiang, A. Chung, and J. Ye, "An active contour model for image segmentation based on elastic interaction," J. Comp. Phys., vol. 219, no. 1, pp. 455-476, 2006.

[58] J. Wang, Y. Guo, Y. Ying, Y. Liu, and Q. Peng, "Fast non-local algorithm for image denoising," in Proc. IEEE Int. Conf. Image Process., 2006, pp. 1429-1432.

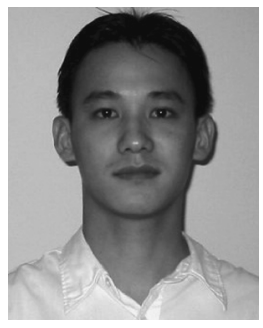

Si Yong Yeo received the B.Eng.and M.Eng. degrees in mechanical and production engineering from Nanyang Technological University, Singapore, in 2002 and 2004, respectively, and is currently pursuing the $\mathrm{Ph} . \mathrm{D}$. degree in the College of Engineering, Swansea University, Swansea, U.K.

His current research interests include image processing, geometry reconstruction, deformable models, shape analysis, and level set methods.

$\mathrm{Mr}$. Yeo is a student member of the European Society of Biomechanics.

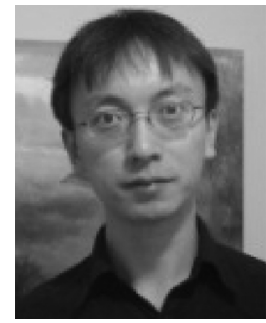

Xianghua Xie (S'03-M'06) received the M.Sc. (with commendation) and Ph.D. degrees in computer science from the University of Bristol, Bristol. U.K. in 2002 and 2006, respectively.

Since 2007, he has been a Lecturer (RCUK Academic Fellow) in the Department of Computer Science, Swansea University, Swansea, U.K. He was previously a Research Associate in the Department of Computer Science, University of Bristol. His current research interests are video analysis, texture analysis, image segmentation, surface inspection, deformable models, and human pose estimation and tracking.

Dr. Xie is a member of the BMVA.

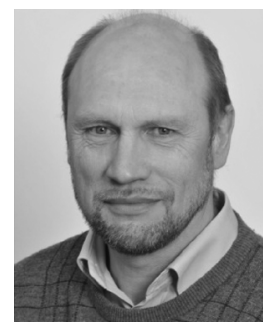

Igor Sazonov received the M.Sc. degree in physics from The Moscow Institute of Physics and Technology, Moscow, Russia, in 1975, and the Ph.D. degree in Physics in from N. N. Andreev Acoustics Institute, Moscow, Russia, in 1983

$\mathrm{He}$ is a Lecturer (RCUK Academic Fellow in Biomedical Engineering) in the College of Engineering, Swansea University, Swansea, U.K. He was previously a Senior Researcher at N. N. Andreev Acoustics Institute (1975-1992) and at A. M. Obukhov Institute of Atmospheric Physics (1992-1999), Moscow, Russia; a full time temporary Lecturer in Applied Mathematics at the University College Cork, Republic of Ireland (1999-2002); Research Assistant at the College of Engineering, Swansea University (2002-2006). His current research interest is hemodynamic and airways patient-specific numerical modeling, including image processing and mesh generation.

Dr. Sazonov is a member of the ESB (European Society of Biomechanics).

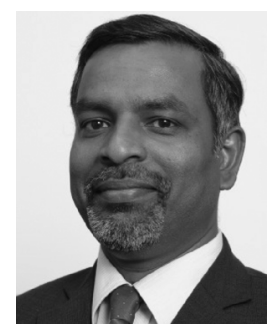

Perumal Nithiarasu received the Ph.D. degree from the Indian Institute of Technology, Madras, India, in 1997, and the D.Sc. degree from Swansea University, Swansea, U.K., in 2007.

$\mathrm{He}$ is currently a Full Professor in the College of Engineering, Swansea University. He was a Reader between 2006 and 2008 and Senior Lecturer between 2004 and 2006, a Lecturer between 2000 and 2004, and a Research Officer between 1996 and 2000 at Swansea University. He currently holds an Advanced Fellowship from the Engineering and Physical Sciences Research Council, UK. His research interests include computational methods and computational bioengineering. He is an editor of the International Journal for Numerical Methods in Biomedical Engineering.

Dr. Nithiarasu is a Fellow of the IMA (U.K.) and IMechE (U.K.) and a Senior Member of the AIAA. 